\title{
Alfnoor: Assessing the Information Content of Ariel's Low-resolution Spectra with Planetary Population Studies
}

\author{
Lorenzo V. Mugnai ${ }^{1}$ (D), Ahmed Al-Refaie ${ }^{2}$ (D), Andrea Bocchieri ${ }^{1}$ (D), Quentin Changeat ${ }^{2}$ (D), Enzo Pascale ${ }^{1}$, and Giovanna Tinetti ${ }^{2}$ (D) \\ ${ }^{1}$ Dipartimento di Fisica, La Sapienza Università di Roma, Piazzale Aldo Moro 2, I-00185 Roma, Italy; lorenzo.mugnai@uniroma1.it \\ ${ }^{2}$ Department of Physics and Astronomy, University College London, Gower Street, London, WC1E 6BT, UK \\ Received 2020 August 24; revised 2021 October 1; accepted 2021 October 8; published 2021 December 3
}

\begin{abstract}
The Ariel Space Telescope will provide a large and diverse sample of exoplanet spectra, performing spectroscopic observations of about 1000 exoplanets in the wavelength range $0.5-7.8 \mu \mathrm{m}$. In this paper, we investigate the information content of Ariel's Reconnaissance Survey low-resolution transmission spectra. Among the goals of the Ariel Reconnaissance Survey is also to identify planets without molecular features in their atmosphere. In this work, (1) we present a strategy that will allow us to select candidate planets to be reobserved in Ariel's higherresolution tier, (2) we propose a metric to preliminary classify exoplanets by their atmospheric composition without performing an atmospheric retrieval, and (3) we introduce the possibility to find other methods to better exploit the data scientific content.
\end{abstract}

Unified Astronomy Thesaurus concepts: Transmission spectroscopy (2133); Exoplanet atmospheric composition (2021); Space telescopes (1547)

\section{Introduction}

In the past decade the number of known exoplanets has increased 10-fold: at the end of 2009 around 400 exoplanets were known, while at the end of 2019 the confirmed discoveries reached more than 4000 . This rapid increase in the exoplanetary science yield is expected to continue, and it will affect not only the number of discovered planets but also our knowledge of planetary formation and evolution. While the discoveries will increase thanks to space missions such as TESS (Ricker et al. 2016), CHEOPS (Cessa et al. 2017), PLATO (Rauer et al. 2014), and Gaia (Gaia Collaboration et al. 2016) and to ground instrumentation such as HARPS (Mayor et al. 2003), HATnet (Bakos 2018), WASP (Pollacco et al. 2006), KELT (Pepper et al. 2018), OGLE (Udalski et al. 2015), NGTS (Wheatley et al. 2013), and many others, our understanding of planets' histories can only grow through planetary composition analysis.

The most effective strategy used today to reveal the atmospheric chemistry and thermodynamics of transiting exoplanets is to use multiband photometry and spectroscopy (e.g., Seager \& Sasselov 2000; Charbonneau et al. 2005; Tinetti et al. 2007; Sing et al. 2016; Huitson et al. 2012; Madhusudhan et al. 2012; Kreidberg et al. 2014; Edwards et al. 2020; Pluriel et al. 2020a; Guilluy et al. 2021; Mugnai et al. 2021). Current instrumentation has enabled this kind of atmospheric characterization for a few tens of exoplanets over a limited wavelength range (e.g., Sing et al. 2016; Tsiaras et al. 2018). To interpret the observed spectra, spectral retrieval techniques, often developed for the study of Earth and solar system planets, have flourished and were adapted to the new field of investigation (e.g., Irwin et al. 2008; Line et al. 2013; Waldmann et al. 2015b; Gandhi \& Madhusudhan 2017; AlRefaie et al. 2021). Most recently, an intense effort has been performed to compare and validate different models developed by

\footnotetext{
Original content from this work may be used under the terms of the Creative Commons Attribution 4.0 licence. Any further distribution of this work must maintain attribution to the author(s) and the title of the work, journal citation and DOI.
}

different teams to assess potential discrepancies among them (Barstow et al. 2020), demonstrating the robustness and consistency of those models.

The Atmospheric Remote-Sensing Infrared Exoplanet Largesurvey, Ariel, will enable the spectroscopic observation of a diverse sample of about 1000 exoplanets (Tinetti et al. 2018; Ariel Definition Study Report ${ }^{3}$ ) in the $0.5-7.8 \mu \mathrm{m}$ wavelength range. The Ariel payload has three photometers (VISPhot, $0.5-0.6 \mu \mathrm{m}$; FGS1, 0.6-0.80 $\mu \mathrm{m}$; FGS2, $0.80-1.1 \mu \mathrm{m})$ and three spectrometers (NIRSpec, $1.1-1.95 \mu \mathrm{m}$ and $R \geqslant 15$; AIRS$\mathrm{CH} 0,1.95-3.9 \mu \mathrm{m}$ and $R \geqslant 100$; AIRS-CH1, 3.9-7.8 $\mu \mathrm{m}$ and $R \geqslant 30$ ). After each observation, the resulting spectrum from each spectrometer is binned during data analysis to optimize the planetary spectrum signal-to-noise ratio $(\mathrm{S} / \mathrm{N})$. Therefore, implementing different binning options, the mission will adopt a four-tier strategy, expected to deliver spectra with different $\mathrm{S} / \mathrm{N}$ to optimize the science return (Tinetti et al. 2018).

Tier 1 was created to deliver a reconnaissance survey where all planets are first observed at low spectral resolution, and only a subset of Tier 1 planets will be further observed to reach $\mathrm{S} / \mathrm{N} \geqslant 7$ at a higher spectral resolution (Tier 2, Tier 3). Tier 1 observations have an $\mathrm{S} / \mathrm{N} \geqslant 7$ when raw spectra are binned into a single spectral point in NIRSpec, two in AIRS-CH0 and one in AIRS$\mathrm{CH} 1$, for a total of four spectral and three photometric data points. For $\sim 50 \%$ of total observed planets, Ariel will provide spectra at Tier 2 resolution. In this tier, raw spectra are binned at $R=10,50$, and 15 in NIRSpec, AIRS-CH0, and AIRS-CH1, respectively, with an $\mathrm{S} / \mathrm{N}$ of 7 or larger. Tier 3 is meant to provide spectra with $\mathrm{S} / \mathrm{N} \sim 7$ for $5 \%-10 \%$ of the total observed targets. In this tier the raw spectral data are binned at $R=20,100$, and 30 in NIRSpec, AIRS-CH0, and AIRS-CH1, respectively. Finally, Tier 4 is conceived for bespoke or phase-curve observations. Among the main goals of Tier 1 observations is to identify planetary spectra that show no molecular absorption features and to select those to be reobserved in the successive tiers.

The aim of this paper is threefold:

\footnotetext{
https://sci.esa.int/web/ariel/-/ariel-definition-study-report-red-book
} 
1. to show the capability of selecting the planets with featureless spectra, which may not be observed again in successive tiers, without involving retrieval techniques;

2. to introduce a metric and show its principal applications as a tool to classify Tier 1 observed planets on their molecular content, to aid in the selection of targets to be reobserved in successive tiers; and

3. to show that other strategies to exploit Ariel Tier 1 data are possible, such as those based on machine learning (ML).

In Section 2 we present our strategy to address these three goals. Our new software, Alfnoor, able to build entire planetary populations, is presented in Section 2.1. Then, we discuss the targets chosen to build the populations and the atmospheric properties used in Section 2.2. In the same section, we also describe a method to identify the flat spectra in the sample (Section 2.3), which is the first paper goal. This method's results are then described in Section 3.1. Then, we describe the metric developed as mentioned in the second goal of this paper (Section 2.4), and we introduce a classification algorithm to compare the metric with (Section 2.4.1). We present in detail the results obtained by our algorithm (Section 3.2), we show the relation between the metric and the input molecular abundances in the planets, and we discuss biases and limitations. Finally, we provide a preliminary assessment of the application of ML and deep-learning techniques to the problem of spectrum classification in Section 2.5, discussing their performance in Section 3.3, but leaving a more thorough investigation to future work. In Section 4 we discuss and compare the results in detail.

\section{Methodology}

\subsection{The Alfnoor Software}

Ariel will provide a sample of hundreds of planetary spectra. To simulate this data set, we develop a new algorithm: Alfnoor, the thousand lights simulator, which was also used for Tier 2 data in Changeat et al. (2020). Alfnoor is a wrapper of TauREx 3 (AlRefaie et al. 2021) and ArielRad (Mugnai et al. 2020). TauREx 3 is a complete rewrite of the atmospheric retrieval code TauREx (Waldmann et al. 2015a, 2015b). ArielRad is the Ariel radiometric model: a software that, given the Ariel payload and mission strategy descriptions, can simulate the signal propagating from a candidate target through the instruments and return the expected instrument noise. ArielRad, therefore, can compute the number of observations needed to match each of the Ariel tier requirements (to reach a minimum $\mathrm{S} / \mathrm{N}=7$ at the tier spectral resolution).

By combining the two software, Alfnoor produces the atmospheric high-resolution forward model of a planet with TauREx 3, bins down the spectrum to the Ariel tier wavelength grid, and adds the expected noise estimated by ArielRad. Consequently, Alfnoor returns a simulation of the planet spectrum as observed in each of the Ariel mission tiers. Iterating this procedure for different planets or compositions, Alfnoor automates the process of building entire planetary populations and therefore a data set that is representative of the one Ariel will provide.

The Alfnoor and the ArielRad tools are not publicly available, currently. However, both TauREx $3^{4,5}$ and a generic radiometric simulator called ExoRad $2.0^{6,7}$ are publicly available on

\footnotetext{
4 https://github.com/ucl-exoplanets/TauREx3_public

5 https://pypi.org/project/taurex/

6 https://github.com/ExObsSim/ExoRad2-public

7 https://pypi.org/project/exorad/
}

Table 1

List of Host Star and Planet Information Obtained from the Ariel Planet Candidate List and Used to Build the Planetary Populations Used in This Work

\begin{tabular}{ll}
\hline \hline Star & Planet \\
\hline Mass & Mass \\
Radius & Radius \\
Effective temperature & Equilibrium temperature \\
Distance & Distance from the star \\
& Orbital period \\
& Transit duration \\
\hline
\end{tabular}

GitHub and PyPI. ArielRad is ExoRad 2.0 configured for the Ariel payload.

\subsection{Planetary Populations}

To build a diverse sample of planets in terms of masses, radii, and temperatures, we use the Ariel candidate list of Edwards et al. (2019). This list contains 1000 planets, selected from both NASA's Exoplanet Archive and TESS-predicted discoveries, and covers a wide range of planetary radii (from $\sim 0.4$ to $\sim 27 \quad R_{\oplus}$ ), masses (from $\sim 0.01$ to $\sim 3000 M_{\oplus}$ ), and equilibrium temperatures (from $\sim 200$ to $\sim 3900 \mathrm{~K}$ ). From that list, we extract the parameters listed in Table 1. Our goal is not to reproduce accurately the composition of the planets in that list, but to test a diverse sample, and therefore we randomly build an atmosphere for each of the listed targets. We produce three planetary populations that will be of use for this work. We call them POP-I, POP-II, and POP-III.

$P O P-I$.-For each planet we randomize the equilibrium temperature, choosing a value between $0.7 \times T_{p}$ and $1.05 \times T_{p}$, where $T_{p}$ is the planet equilibrium temperature in Edwards et al. (2019). This randomization is biased toward lower temperature values as we probe the terminator region, where the spectral features are affected by both the dayside and nightside temperatures (Caldas et al. 2019; Pluriel et al. 2020b; Skaf et al. 2020). The temperature randomization range is consistent with the work presented in Changeat et al. (2020).

Then, for each planet we consider an isothermal temperature - pressure profile; we add a constant vertical chemical profile (Moses et al. 2011) for every molecule from a list of selected molecules (the abundances are randomized according to defined boundaries). Finally, we add randomly generated gray opaque clouds. We use the plane-parallel approximation, building 100 plane-parallel layers to uniformly sample in logspace the pressure range $10^{-4}-10^{6} \mathrm{~Pa}$. Every atmosphere is built with randomized relative abundances of $\mathrm{CH}_{4}, \mathrm{H}_{2} \mathrm{O}, \mathrm{CO}_{2}$, and $\mathrm{NH}_{3}$ on a uniform logarithmic scale between $10^{-7}$ and $10^{-2}$. Such a large range allows us to explore the sensitivity of our developed method to very different abundances. We also randomized the cloud surface pressure varying between $5 \times 10^{2}$ and $10^{6} \mathrm{~Pa}$, similarly to what is presented in Changeat et al. (2020), to explore the whole range from overcast to cloud-free atmospheres, respectively. Using these boundaries, we obtain that $\sim 40 \%$ of the atmospheres in the populations contain clouds to at least $10^{4} \mathrm{~Pa}$ (surface pressure), as expected from Tsiaras et al. (2018) and Iyer et al. (2016). Every planet is considered filled with an $\mathrm{H}_{2}$ and $\mathrm{He}$ atmosphere with mixed ratio $\mathrm{He} / \mathrm{H}_{2}=0.17$. A list of the opacities used in this work is reported in Table 2.

As already mentioned, following the aims of this paper, we do not focus on the consistency of the atmospheric models used 


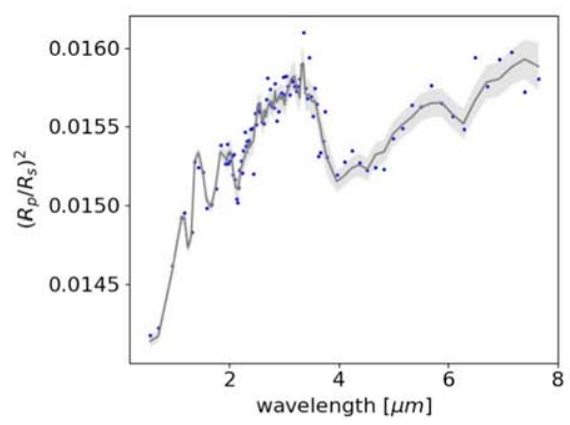

(a) HD 209458b -like planet.

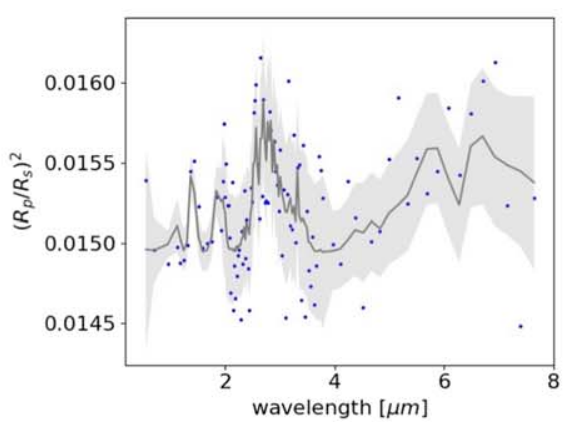

(b) GJ 1214b -like planet.

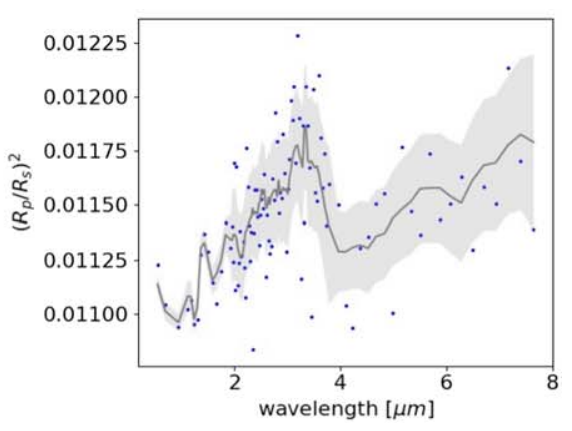

(c) WASP 79b -like planet.

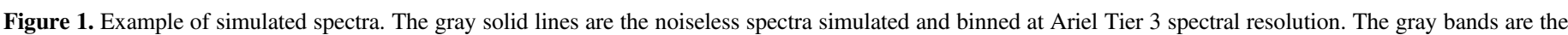

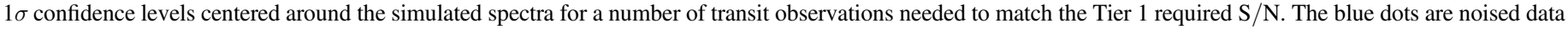

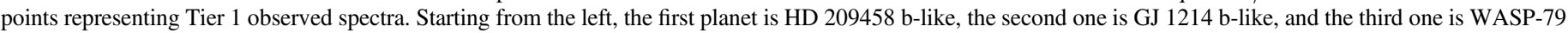
b-like. Their atmospheres are built as described in Section 2.2.

Table 2

List of Opacities Used in This Work and Their References

\begin{tabular}{ll}
\hline \hline Opacity & Reference \\
\hline $\mathrm{H}_{2}-\mathrm{H}_{2}$ & Abel et al. (2011), Fletcher et al. (2018) \\
$\mathrm{H}_{2}-\mathrm{He}$ & Abel et al. (2012) \\
$\mathrm{H}_{2} \mathrm{O}$ & Barton et al. (2017), Polyansky et al. (2018) \\
$\mathrm{CH}_{4}$ & Hill et al. (2013), Yurchenko \& Tennyson (2014) \\
$\mathrm{CO}_{2}$ & Rothman et al. (2010) \\
$\mathrm{NH}_{3}$ & Yurchenko et al. (2011), Tennyson \& Yurchenko (2012) \\
\hline
\end{tabular}

to build the population. The spectra generated will only be used as "transmission spectral shapes" to test our methods against. No information other than the planet transmission spectrum is used in this work.

Each planetary spectrum generated by Alfnoor is binned at Ariel's Tier 3 spectral resolution. These spectra make up the "noiseless spectra" data set. ArielRad then predicts the noise for each spectral bin at the tier resolution. To reproduce a Tier 1 observation, we scatter the data around the true value according to a normal distribution with the mean coinciding with the simulated spectrum and a standard deviation equal to the noise estimated with ArielRad at each spectral bin. This noise is a rescaled version of the Tier 3 noise, obtained by combining the number of transit observations needed to match the Tier 1 required $\mathrm{S} / \mathrm{N}$. Using these scattered spectra, we build the "observed spectra" data set. Examples of the resulting spectra are shown in Figure 1.

We generate POP-I using the full 1000-planet candidate list and produce one realization for each planet. A similar approach was used by Changeat et al. (2020) in their investigation of the Ariel Tier 2 observations. We use the POP-I population to test the strategies described later in the text.

POP-II.-We produce another data set, keeping the same 1000 planets from the target list and the randomization rules of POP-I. However, this time we modify the chemical composition to include only $\mathrm{H}_{2} \mathrm{O}$ and $\mathrm{CH}_{4}$. We use POP-II to perform tests against a simpler population, as detailed later in the text.

POP-III.-To build the last population, we use the same list of 1000 planets, where each planet is repeated four times, such that there are four randomized atmospheres for each unique set of stellar and planetary properties that defines a planet. While the temperature and cloud conditions used are the same as those discussed for POP-I, for each molecule we widen the abundance boundaries to $10^{-9}-10^{-2}$ on a uniform logarithmic scale. We call this population POP-III, and we use it to train our ML algorithms.

\subsection{Flat Planet Detection}

The first goal of this work, as listed in Section 1, is to identify featureless spectra. This will help in the selection of targets to be reobserved in Ariel's higher tiers. Given the property of the Ariel payload, we divide the spectral wavelength range into four parts or bands:

1. from 0.5 to $1.1 \mu \mathrm{m}$, sampled by three photometers;

2. from 1.1 to $1.95 \mu \mathrm{m}$, corresponding to the NIRSpec wavelength range;

3. from 1.95 to $3.9 \mu \mathrm{m}$, corresponding to the AIRS-CH0 wavelength range; and

4. from 3.9 to $7.8 \mu \mathrm{m}$, corresponding to the AIRS-CH1 wavelength range.

For every planet and for every band we estimate a $\chi^{2}$ using all measurements in the band to assess the compatibility with a flat, zero-gradient line: for each planet there are four $\chi^{2}$ estimates, one for each band above. We reject the hypothesis of spectral flatness in a given band with a $3 \sigma$ confidence if $\chi^{2}>1+3 \sqrt{\frac{2}{\nu}}$, where $\nu$ are the degrees of freedom. Therefore, if any of the four bands has a $\chi^{2}$ smaller than this number, we mark the band as flat. If a planetary spectrum has all four bands marked as flat, it is classified as a flat spectrum. This strategy is similar to that presented in Zellem et al. (2019); however, while in that work the authors were only focused on the Ariel FGS channels, here we are considering the full Ariel spectral coverage.

\subsection{An Optimized Molecular Metric}

The second goal listed in Section 1 is to develop a metric, $M_{\text {mol }}$, to assess the presence of a molecule, mol, in the planet's atmosphere. We want this metric to work in such a way that by comparing two molecules the metric produces a diagram similar to that in Figure 2. In the diagram we can distinguish four regions: two regions where the atmospheres are rich in a single molecule and therefore only show its characteristic features, a third region where the atmospheres show features from both molecules, and a fourth region where features are absent, either because the planets have flat spectra or because 


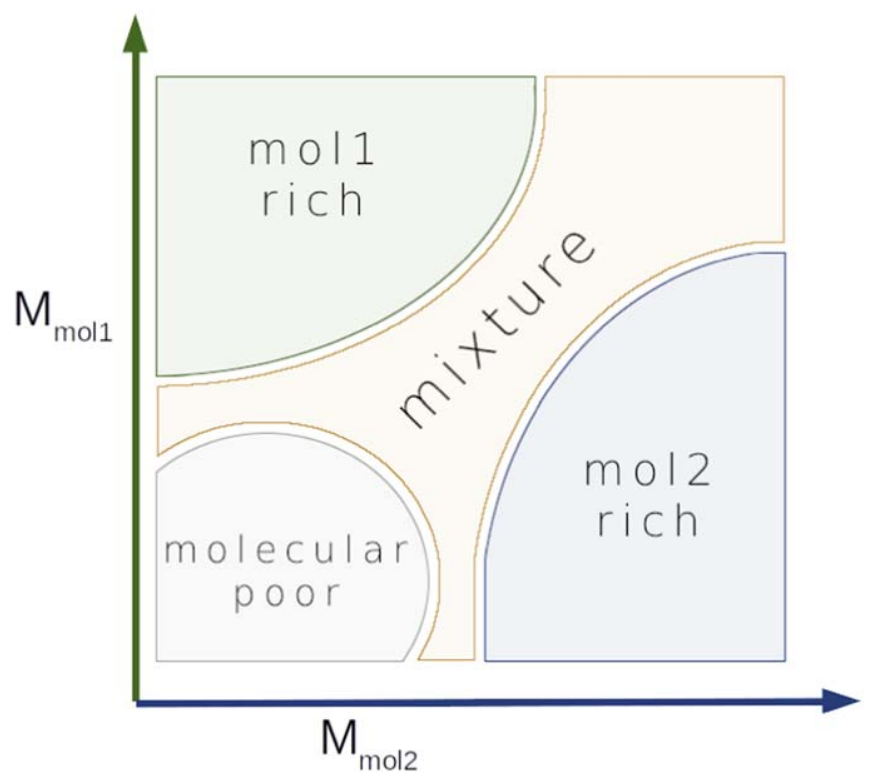

Figure 2. Illustration of the diagram we expect to build with our metric. Here, the metric is used to compare two molecules, moll and mol2. By drawing $M_{\text {mol1 }}$ versus $M_{\text {mol2 }}$, we aim to separate four different regions: one rich in the first molecule at the top left (green), where $M_{\text {moll }}$ grows and $M_{\text {mol2 }}$ is low; a similar region at the bottom right (blue), where the planet atmosphere is rich in the second molecule, because $M_{\mathrm{mol} 2}$ is high and $M_{\text {mol1 }}$ is low; a region where molecular-poor planets are located (gray), or those that have no features in the considered bands, where both $M_{\mathrm{mol1}}$ and $M_{\mathrm{mol} 2}$ are low; and a region for mixed atmosphere (yellow) in the central portion of the diagram.

the features from both molecules do not emerge from a thick layer of clouds.

To compare different planets and constrain their atmospheric molecular content, the metric should be (i) sensitive to the spectral signature of molecules, (ii) independent of the planet size, and (iii) independent of the scale height. Here we present a metric that fulfills these three conditions and show its current limitations.

For each molecule, we select $N$ bands within the Ariel wavelength range, where the molecular features in the transmission spectrum are strong. Then, for each planet, we compute the average in each band, $S_{\text {band }_{i}}$, and its dispersion, $\sigma_{\text {band }_{i}}$ :

$$
\begin{gathered}
S_{\text {band }_{i}}=\frac{1}{M} \sum_{j}^{M} S_{j} \\
\sigma_{\text {band }_{i}}=\sqrt{\frac{1}{M} \sum_{j}^{M}\left(S_{j}-S_{\text {band }_{i}}\right)^{2}},
\end{gathered}
$$

where $M$ is the number of spectral bins in the band and $S_{j}$ is the atmospheric transmission spectrum estimated in the $j$ th wavelength bin.

We do the same with a control band where we know that there are no major molecular features from the molecule considered, called the "normalization band," obtaining $S_{\text {norm }}$ and $\sigma_{\text {norm. }}$. We select a different normalization band for each molecule (Table 3).

Thus, for each molecule, mol, we define

$$
M_{\mathrm{mol}}=\frac{1}{N} \sum_{i}^{N} \frac{S_{\mathrm{band}_{i}}-S_{\mathrm{norm}}}{\sqrt{\sigma_{\mathrm{band}_{i}}^{2}+\sigma_{\text {norm }^{2}}^{2}}} .
$$

Table 3

Wavelength Ranges Used to Select the Molecular Features in the Spectra (Top) and the Normalization Bands (Bottom) for $\mathrm{H}_{2} \mathrm{O}, \mathrm{CH}_{4}$, and $\mathrm{CO}_{2}$

\begin{tabular}{lll}
\hline \hline $\mathbf{H}_{2} \mathbf{O}$ & $\mathbf{C H}_{4}$ & $\mathbf{C O}_{2}$ \\
\hline $1.2-1.6 \mu \mathrm{m}$ & $1.5-1.8 \mu \mathrm{m}$ & $1.9-2.3 \mu \mathrm{m}$ \\
$1.7-2.1 \mu \mathrm{m}$ & $2.1-2.6 \mu \mathrm{m}$ & $2.6-3.2 \mu \mathrm{m}$ \\
$2.6-3.0 \mu \mathrm{m}$ & $3.1-3.7 \mu \mathrm{m}$ & $4.2-4.8 \mu \mathrm{m}$ \\
$5.4-6.1 \mu \mathrm{m}$ & & \\
$6.5-7 \mu \mathrm{m}$ & & \\
\hline Molecule & & Normalization \\
\hline $\mathrm{H}_{2} \mathrm{O}$ & & $3.6-4.2 \mu \mathrm{m}$ \\
$\mathrm{CH}_{4}$ & & $4.0-5.0 \mu \mathrm{m}$ \\
$\mathrm{CO}_{2}$ & & $5.0-6.0 \mu \mathrm{m}$ \\
\hline
\end{tabular}

Defined in this way, $M_{\mathrm{mol}}$ is similar to an $\mathrm{S} / \mathrm{N}$, where the signals are the molecular features arising above the "normalization band" and the noise is the dispersion in the band. Therefore,

$$
\sigma_{M_{\mathrm{mol}}}=\frac{1}{\sqrt{N}}
$$

The metric thus designed, by averaging the contribution of $N$ different bands, corresponding to $N$ different features of the same molecule, reduces the chance to be misled by overlapping features in one of the bands considered. As Ariel's Tier 1 is optimized for low-resolution spectroscopy, spectral binning increases the $\mathrm{S} / \mathrm{N}$. Also, this metric is (i) sensitive to the presence of molecules, (ii) independent of the planet size, and (iii) independent of the scale height (see the Appendix for details), at the cost of the introduction of a bias: Equation (2) provides an estimate of the spectral dispersion when applied to noiseless spectra, and it is larger for observed spectra because of the presence of measurement noise. Therefore, the absolute value of $M_{\text {mol }}$ of Equation (3) is always smaller on observed spectra compared to noiseless spectra of the same planet. While the bias effects are further discussed in Section 4.1, we note here that a detailed characterization of the instrumental noise would allow us to debias the metric, but we leave this investigation to future work, and we focus the attention on the performance of the metric in extracting information from Tier 1 observations.

To maximize the metric efficiency, the challenge is to identify the best-performing wavelength range to use: large enough to reduce the uncertainty introduced by the observational noise, but small enough to distinguish the molecular features of interest.

In this work, we consider only $\mathrm{H}_{2} \mathrm{O}, \mathrm{CH}_{4}$, and $\mathrm{CO}_{2}$, and the bands used are listed in Table 3. Even though $\mathrm{NH}_{3}$ is present in our sample, it is used only to introduce a nuisance and challenge our metric, because $\mathrm{NH}_{3}$ has features overlapping with those of water. We use three feature bands for $\mathrm{CH}_{4}$ and $\mathrm{CO}_{2}$ and five for $\mathrm{H}_{2} \mathrm{O}$. Examples of the bands used for $\mathrm{CH}_{4}$ and $\mathrm{H}_{2} \mathrm{O}$ are shown in Figure 3, where, for the same planetary template, HD 209458 b, we simulate different atmospheres (overcast, $\mathrm{CH}_{4}$ rich, and $\mathrm{H}_{2} \mathrm{O}$ rich) to show how the metric captures the relevant spectroscopic features.

In the next section, we show how we intend to use this metric to build a diagram similar to that of Figure 2. 


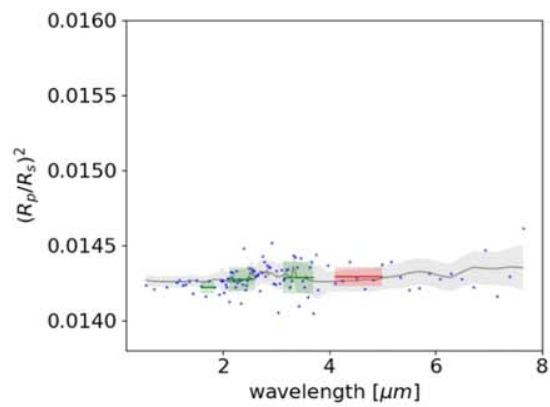

(a) Overcast HD 209458b -like planet with $M_{\mathrm{CH}_{4}}$ data bands highlighted.

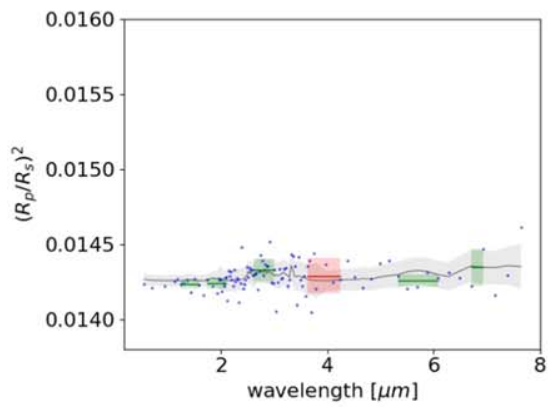

(d) Overcast HD 209458b -like planet with $M_{\mathrm{H}_{2} \mathrm{O}}$ data bands highlighted.

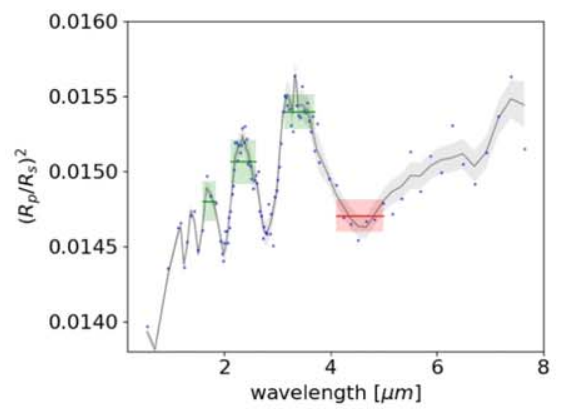

(b) Methane rich HD 209458b like planet with $\mathrm{MCH}_{4}$ data bands highlighted.

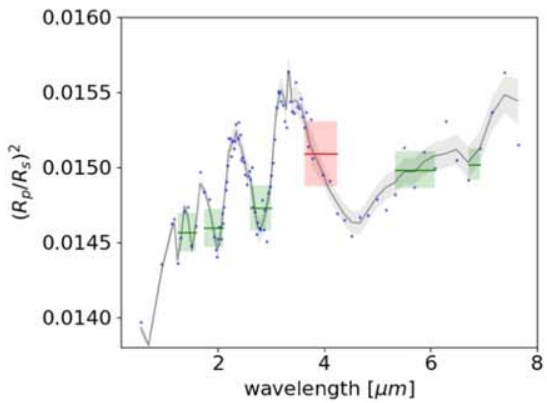

(e) Methane rich HD 209458b like planet with $M_{\mathrm{H}_{2} \mathrm{O}}$ data bands highlighted.

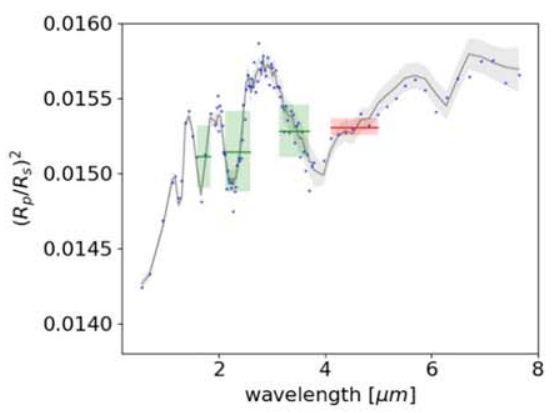

(c) Water rich HD 209458b -like planet with $\mathrm{MCH}_{4}$ data bands highlighted.

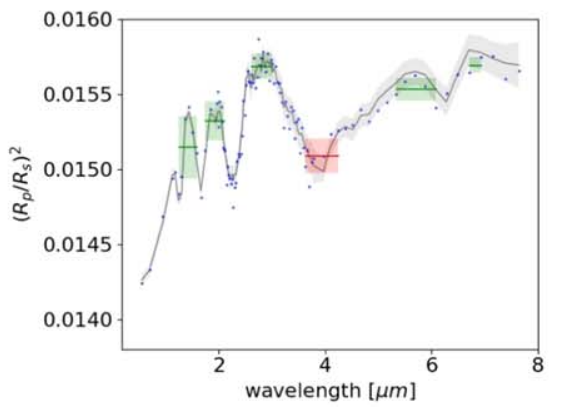

(f) Water rich HD 209458b -like planet with $M_{\mathrm{H}_{2} \mathrm{O}}$ data bands highlighted.

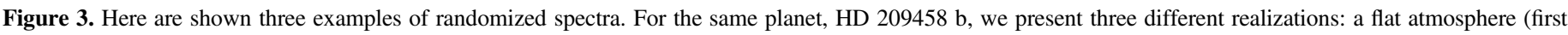

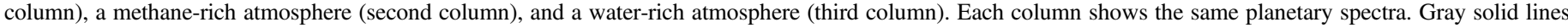

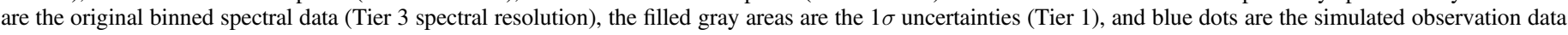

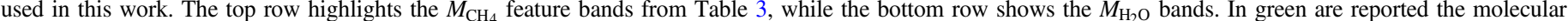

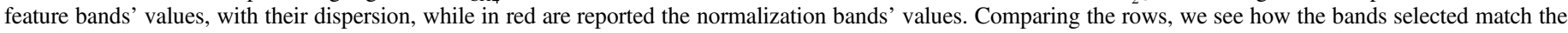
relevant molecular spectral features.

\subsubsection{Planets Classification}

The metric is required to be calibrated to assess its capability to estimate the presence of a molecule. The final product is a diagram similar to Figure 2, which can be used as a look-up table, such that, given an observed spectrum, its corresponding $M_{\text {mol }}$ can be located on the diagram and its possible composition inferred.

To assess the ability of the metric to separate the atmospheres in the sample, we use the $k$-nearest neighbors (KNN) algorithm, a nonparametric pattern recognition algorithm (Hastie et al. 2009). This algorithm, after a training process, assigns a class to an element given the properties of its neighbors. The goal is to classify observed spectra by their molecular content, according to their $M_{\text {mol }}$. Considering two molecules at a time, we first define four classes of planets: molecular poor, moll rich, mol 2 rich, and mixture, as defined in Table 4.

The KNN algorithm used classifies each planet according to the $20(k=20)$ nearest planets, in the $M_{\text {mol1 }}$ versus $M_{\text {mol2 }}$ space, in the same data set. We choose to use 20 neighbors (2\% of the full data set) to minimize the number of misclassified planets. The closest neighbors are uniformly weighted, and we verified that weighting the neighbors with their Euclidean distance in the metric space does not affect the results significantly.
Table 4

Diagram Classes and Conditions

\begin{tabular}{ll}
\hline \hline Class & Condition \\
\hline Molecular poor & $A b_{\text {mol1 }}<10^{-5}$ and $A b_{\text {mol2 }}<10^{-5}$ \\
Mol1 rich & $A b_{\text {mol1 }}>10^{-4}$ and $A b_{\text {mol1 }}>10 \times A b_{\text {mol2 }}$ \\
Mol2 rich & $A b_{\text {mol2 }}>10^{-4}$ and $A b_{\text {mol2 }}>10 \times A b_{\text {mol1 }}$ \\
Mixture & everything else
\end{tabular}

The analysis involves three separated steps, summarized in Figure 4, applied to POP-I.

Step 1.-We estimate the $\left(M_{\mathrm{mol1}}, M_{\mathrm{mol} 2}\right)$ on the POP-I observed spectra. We assign a class to each POP-I planet using its input molecular abundance values, $A b_{\text {mol }}$, which are stored during the population production. This process is described in the top branch of Figure 4.

Step 2.-To calibrate the metric, we map the metric space grid by training the KNN algorithm on the $\left(M_{\mathrm{mol} 1}, M_{\mathrm{mol} 2}\right)$ estimated from the noiseless POP-I planetary spectra. We assign again a class to each planet using its input molecular abundance, $A b_{\mathrm{mol}}$, and the training is performed on a randomly chosen selection accounting for $70 \%$ of the data set, while we use the remaining $30 \%$ to test the success of the training. Finally, we classify each point $\left(M_{\mathrm{mol1}}, M_{\mathrm{mol} 2}\right)$ of the $M_{\mathrm{mol}}$ space grid $M_{\text {mol }}$ sampled at a step width of $0.2 M_{\text {mol }}$, obtaining 


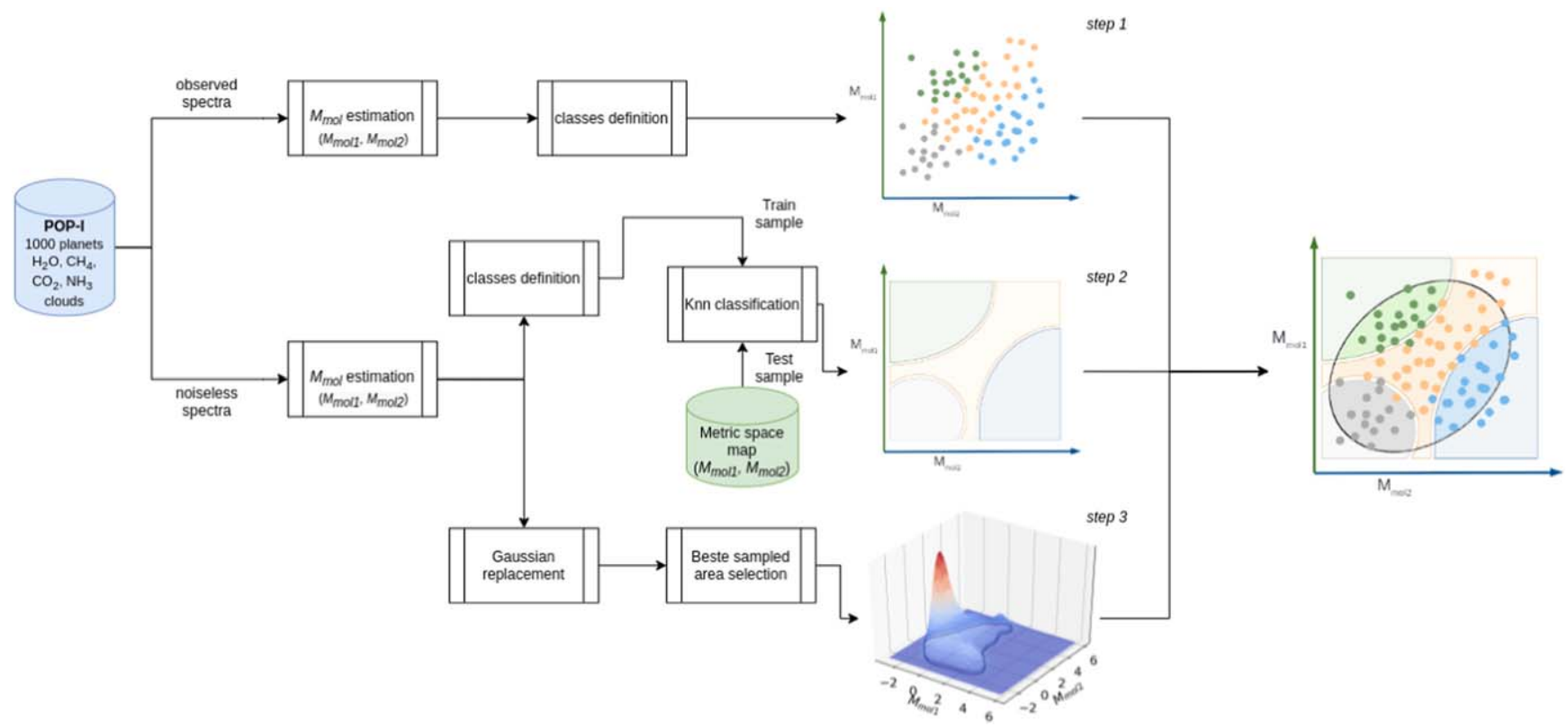

Figure 4. Planet classification summary. The figure reports the steps implemented to build the diagram in Figure 2. Starting from POP-I, for each planet we compute $\left(M_{\mathrm{mol1}}, M_{\mathrm{mol} 2}\right)$ for the considered molecules and for both observed and noiseless data. Following the top branch, classes are assigned to the observed spectra (step 1 in the text). Following the middle branch, a KNN classification is performed on noiseless spectra to calibrate the metric space (step 2 in the text). Following the bottom branch, the distribution of noiseless metric data points is convolved with a 2D Gaussian with varying widths to generate a unit-normalized volume. The intersection between this volume and the calibration of step 2 selects the best-sampled (i.e., calibrated) region in the metric space (step 3 in the text). The combination of these three steps is shown in the rightmost diagram to be compared with Figure 2.

a map comparable to Figure 2. This part of the procedure corresponds to the central branch of Figure 4.

Step 3.-Since the noiseless planetary spectra are not expected to sample the parameter space uniformly, we build a mask to select a region of the $\left(M_{\mathrm{mol} 1}, M_{\mathrm{mol} 2}\right)$ space that is sufficiently well sampled to achieve a reliable classification. To do so, we replace each $\left(M_{\text {mol1 }}, M_{\text {mol2 } 2}\right)$ point representing a noiseless planetary spectrum with a two-dimensional Gaussian distribution using the metric dispersion in the two directions as $\sigma$. We sum the Gaussian volumes on the parameter space, ending up, after volume normalization, with a statistical distribution of our data points on the parameter space grid. Then, we select a region in the metric space that results in a total volume of $95 \%$, therefore removing all undersampled areas from the grid. This last step is represented in the bottom branch of Figure 4.

The combination of the three steps is shown in the rightmost panel of Figure 4, and it is the equivalent of Figure 2 calibrated for the metric on the investigated population.

\subsection{Deep and Machine Learning}

The metric presented in Section 2.4 is based on binning the spectra and therefore is equivalent to using Ariel as a multiband photometer. This strategy is in line with the Tier 1 definition of Tinetti et al. (2018). However, we are also investigating different strategies to classify spectra by their molecular content (third goal listed in Section 1). Deep-learning and ML techniques are promising because these algorithms can learn to classify planets from their spectral shape over the whole wavelength range sampled by Ariel. Another advantage over the metric is that ML techniques are not supposed to be biased by the instrumental noise, or at least they can be made to learn how to deal with the bias provided that a sufficiently large and representative set of examples is provided in training. To train the algorithms, we use the POP-III observed spectra and their known abundances as a training sample. Each example spectrum is normalized to zero mean and unit dispersion. The normalization facilitates the training process but might introduce a bias that may be very similar to that affecting the metric. A detailed investigation of these aspects concerning ML is left to future work. Knowing the input abundance of each planet, $A b_{\mathrm{mol}}$, we can define a threshold and flag a planet as bearing a certain molecule if $A b_{\text {mol }}$ is larger than the threshold. This means that, for each molecule, the algorithm learns to flag the planets as bearing that molecule by looking at characteristic spectral shapes. Then, we measure the algorithm's ability to "learn" by how much they can generalize their predictions to unknown shapes, testing it on POP-I observed spectra, used as a test data set. The comparison of the ML classification with the known input abundance of each POP-I planet provides an estimate of the success rate.

A detailed investigation of the use of these algorithms and their limitations will be discussed in future work: here we report only an example of how these tools might be used, and we compare some preliminary results with the outcomes of the metric of Section 2.4. We implemented all algorithms in Python using the scikit-learn ${ }^{8}$ package presented in Pedregosa et al. (2011).

The first ML algorithm we use is the KNN algorithm described above. This time we want to simply classify the planets and not to produce a map as in Section 2.4.1. For this exercise, we use the scikit-learn default KNN setting: $k=5$ and uniform weight for the neighbors. Other ML algorithms can be used to classify planets. Here we also present our preliminary results using a Multi-layer Perceptron (MLP) classifier, a Random Forest Classifier (RFC), and a Support Vector Classifier (SVC; e.g., Goodfellow et al. 2016; Sturrock et al. 2019). The

\footnotetext{
8 https://scikit-learn.org/0.22/
} 


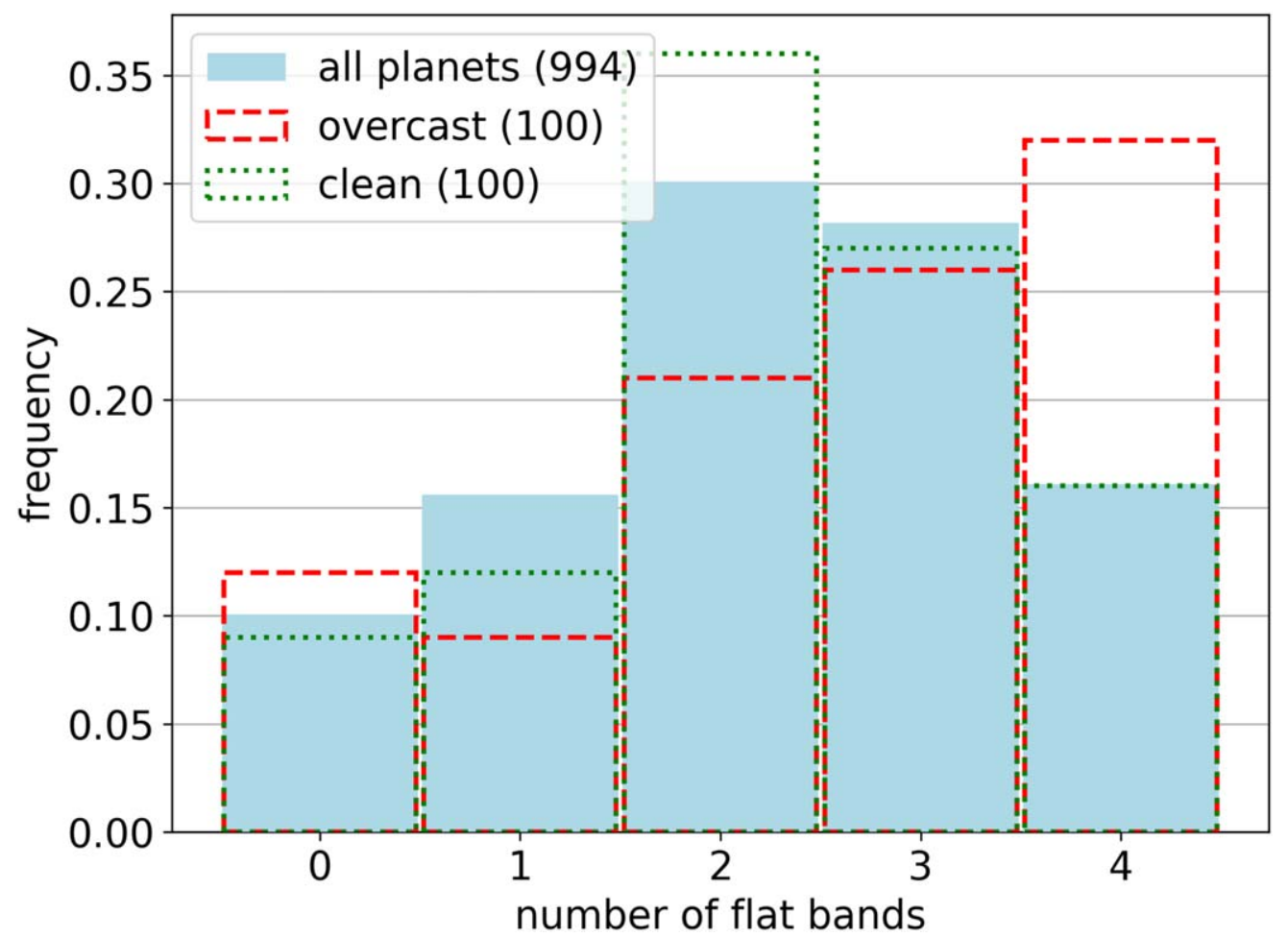

Figure 5. The histograms show the frequency of planets in the population vs. the number of flat bands. We consider four bands: one for the photometers (VisPhot, FGS1, FGS2) and one for each spectrometer (NIRSpec, AIRS-CH0, and AIRS-CH1). Each band is compared with a constant value using a $\chi^{2}$ test to determine its compatibility with flatness. The light-blue histogram shows the frequency of planets in the POP-I population with flat bands. The red dashed histogram shows the same statistic but for a selection of the 100 planets of POP-I that are more overcast. The green dotted histogram shows the opposite situation, for a selection of the 100 planets in POP-I for which the cloud pressure surface is the lowest (see text for details). We notice that the overcast planets show more flat bands than planets with fewer clouds.

MLP is a feed-forward neural network composed of multiple layers of perceptrons largely used in classification problems. To produce the results shown later in the text, we use an MLP network keeping the scikit-learn default settings (a single hidden layer made of 100 units), and we classify the spectra with the same procedure used for the KNN. The RFC is an ensemble of decision trees used for classification, where each decision tree is a directed graph and each vertex is a binary test. In this work, we use an RFC setup commonly used in binary decision problems, which has a number of features equal to the square root of the number of input data points because, for scikit-learn, this is the default configuration. The SVC is a Support Vector Machine method, a family of nonprobabilistic linear classifiers that construct hyperplanes to separate the data points. For the aim of this paper, we implemented a simple SVC shaping the decision function in "one-versus-one" mode, as it is the default configuration in scikit-learn at the moment of writing.

\section{Results \\ 3.1. Identifying Flat Spectra}

Shown in Figure 5 is the frequency of observed planets in the POP-I population that have a certain number of flat bands. In this population, $16 \%$ of planets are to be considered "flat," as all of the four spectral bands considered are flat. From the figure, we notice that around $46 \%$ of the planets in the population have three or more flat bands, which is consistent with POP-I known properties and with the ground truth (Iyer et al. 2016; Tsiaras et al. 2018), as mentioned in Section 2.2. In the same figure the same statistic is shown for the 100 planets of POP-I most covered in clouds (corresponding to a cloud surface pressure of roughly $<10^{3} \mathrm{~Pa}$ ) and for the 100 planets of POP-I with fewer clouds (corresponding to a cloud surface pressure of roughly $>10^{5.5} \mathrm{~Pa}$ ). This comparison shows how overcast planets averagely present more flat bands than clean planets, demonstrating how this approach is sensitive to the presence of clouds.

This result clearly shows that Tier 1 observations are effective in the identification of atmospheres with no detectable molecular absorption features.

\subsection{Spectra Classification}

The $M_{\text {mol }}$ (Section 2.4) estimated for the observed POP-I planets are shown in Figure 6 for different pairs of molecules: $\mathrm{CH}_{4}-\mathrm{CO}_{2}$ and $\mathrm{CH}_{4}-\mathrm{H}_{2} \mathrm{O}$. Comparing the top left and right panels in Figure 6, we notice from the color scale that our metric can separate between planets bearing more or less methane (dark- and light-green dots, respectively) or carbon dioxide (dark- and lightorange dots, respectively). The bottom panels, and the bottom right panel in particular, show that it is harder to separate planets bearing more or less water (dark- and light-blue dots, respectively). Water data appear more clustered around the axes' origin than the top row, and the water colored data points are not as clearly separated according to their color gradient as the methane or the carbon dioxide data points are. A possible explanation is that $\mathrm{CH}_{4}$ and $\mathrm{CO}_{2}$ have strong spectral features, with isolated transmission features in the range $3-4 \mu \mathrm{m}$ and $4-5 \mu \mathrm{m}$, respectively, while $\mathrm{H}_{2} \mathrm{O}$ features are less obvious and frequently overlap with the ones of $\mathrm{NH}_{3}$, which is present in the population (Tinetti et al. 2013). An alternative explanation is that involving a bias in the metric affects more strongly the water bands. 


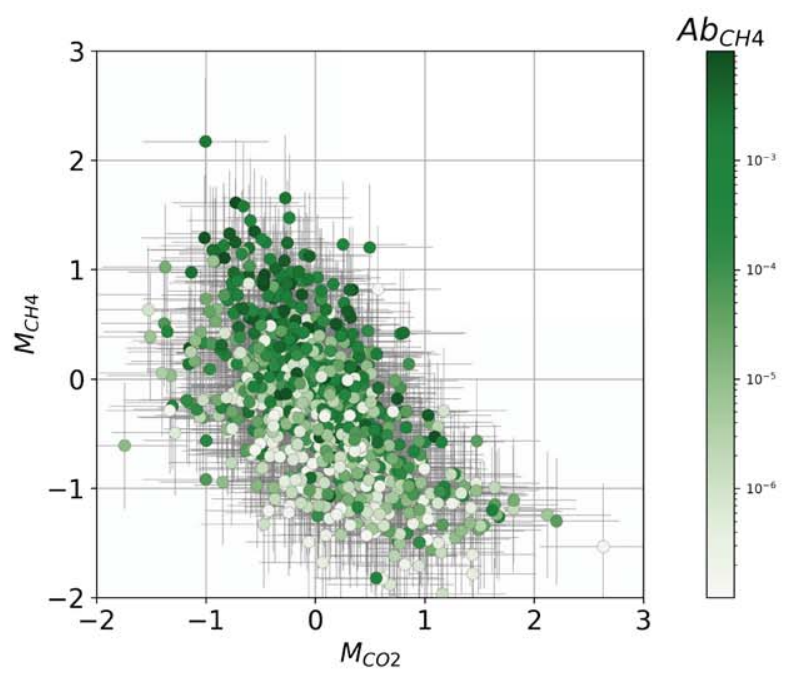

(a) POP-I estimated $M_{\mathrm{CO}_{2}}-M_{\mathrm{CH}_{4}}$ coloured by $\mathrm{CH}_{4}$.

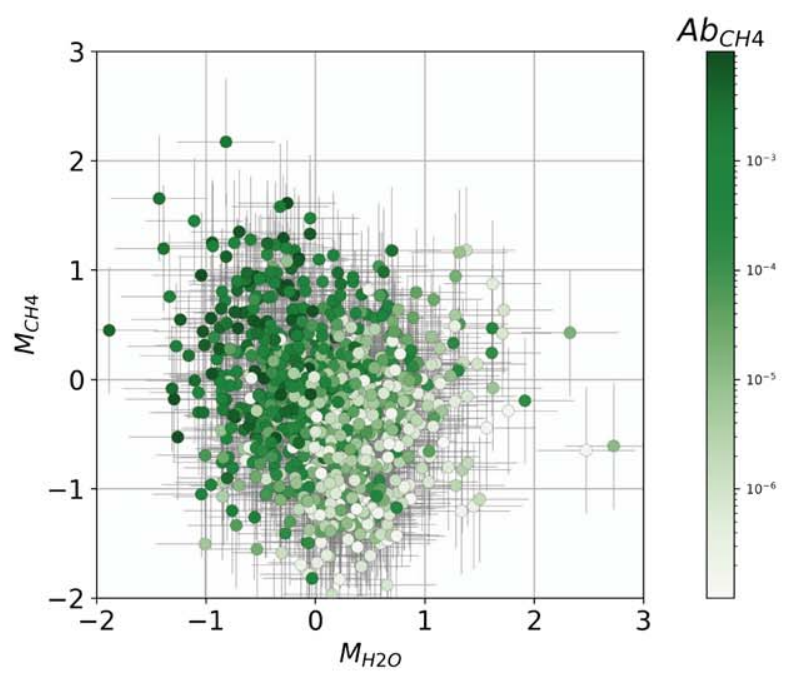

(c) POP-I estimated $M_{\mathrm{H}_{2} \mathrm{O}}-M_{\mathrm{CH}_{4}}$ coloured by $\mathrm{CH}_{4}$.

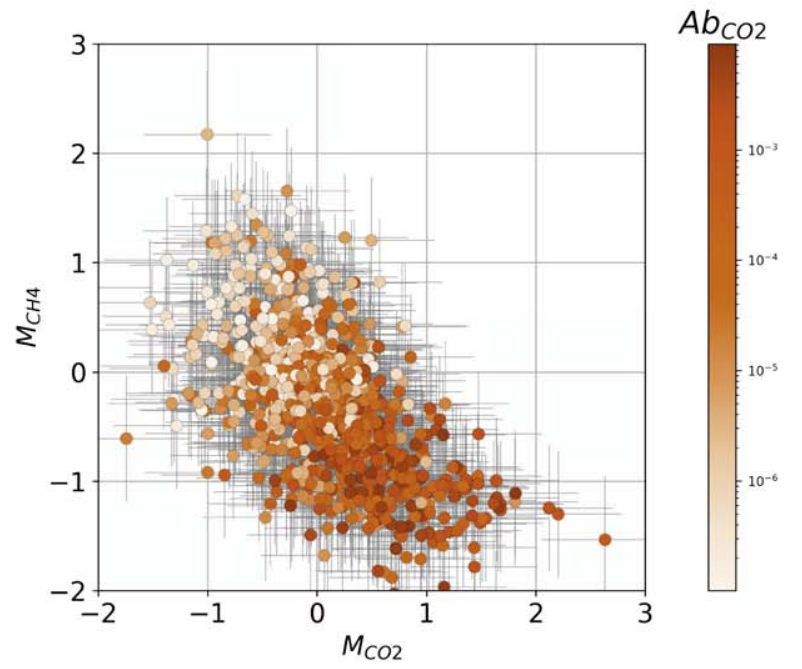

(b) POP-I estimated $M_{\mathrm{CO}_{2}}-M_{\mathrm{CH}_{4}}$ coloured by $\mathrm{CO}_{2}$.

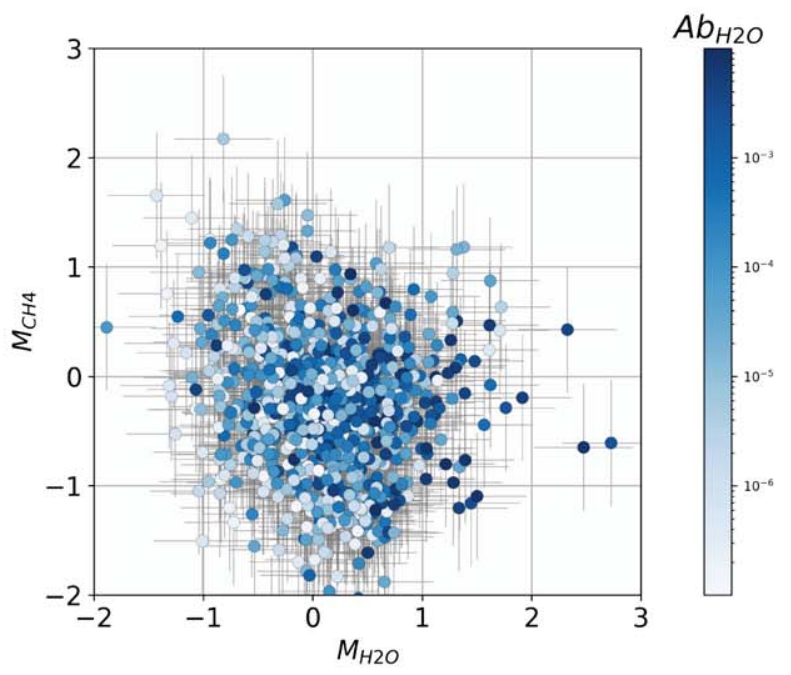

(d) POP-I estimated $M_{\mathrm{H}_{2} \mathrm{O}}-M_{\mathrm{CH}_{4}}$ coloured by $\mathrm{H}_{2} \mathrm{O}$.

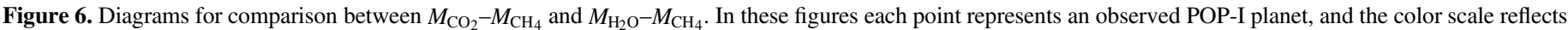

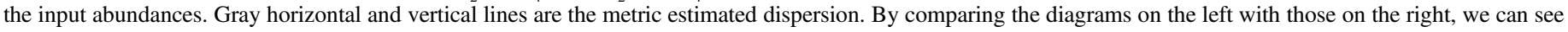
that planets bearing more $\mathrm{CH}_{4}$ are located on the top left, while the ones bearing more $\mathrm{CO}_{2}$ and $\mathrm{H}_{2} \mathrm{O}$ are on the bottom right.

The diagrams of Figure 6 are reproduced in Figure 7, where the data points are now color-coded following the assigned classes (step 1, Section 2.4.1), and the background colors, constructed by training the KNN on noiseless spectra (steps 2 and 3, Section 2.4.1), serve as reference and calibrated regions in the metric space. It can be noticed that the metric has the desired response from the similarities between the reference regions in Figure 7 and those of Figure 2, with a clear separation in the metric space. The data points tend to cluster toward the origin of the grid more strongly than the reference regions. This is the effect of the bias, further discussed in Section 4.1.

Figure 8 shows the relation between the metric, $M_{\mathrm{mol}}$, estimated on POP-I observed spectra, and the input abundances, $A b_{\text {mol. }}$. The coefficients of the linear trends of $M_{\text {mol }}$ versus the logarithm of $A b_{\text {mol }}$ are listed in Table 5. An appreciable trend is detected with $\log$ abundances of $\mathrm{CO}_{2}$ and $\mathrm{CH}_{4}$, while the $\mathrm{H}_{2} \mathrm{O}$ metric shows only a weak trend with input log abundance. Anticorrelations between, e.g., $M_{\mathrm{CH}_{4}}-\log \left(A b_{\mathrm{CO}_{2}}\right)$ or $M_{\mathrm{H}_{2} \mathrm{O}}-\log \left(A b_{\mathrm{CH}_{4}}\right)$ are present as we are considering juxtaposed bands to size these molecules, as listed in Table 3. The logarithmic abundances of $\mathrm{H}_{2} \mathrm{O}$ and $\mathrm{NH}_{3}$ show similar correlations to $M_{\mathrm{H}_{2} \mathrm{O}}$. While this is expected, as the two molecules manifest similar spectral shapes, the water sensitivity of the metric to the abundance may also be limited by the noise, by a bias squeezing the metric to small values, or both, and further investigation is required in future work. However, the metric is an estimator for the classification of atmospheres on the basis of their molecular content, and it would be misleading to expect the metric to provide robust estimates of abundances, for which spectral retrieval techniques are more appropriate. These aspects are further discussed in Section 4.2, as well as in Section 4.4, where we show with an example how a retrieval exercise is effective in constraining the input abundances of the molecules considered, water included. 


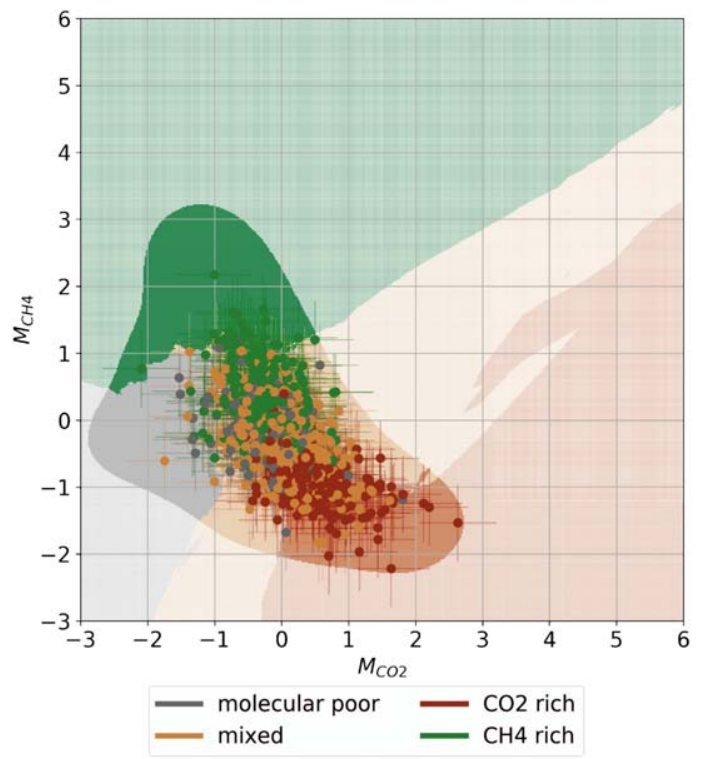

(a) $M_{\mathrm{CO}_{2}}-M_{\mathrm{CH}_{4}}$ - observed spectra.

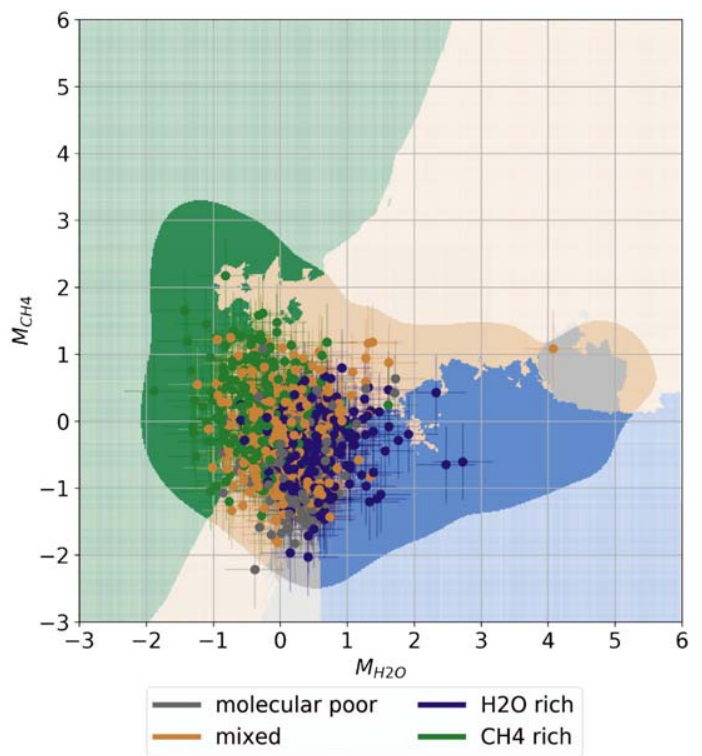

(b) $M_{\mathrm{H}_{2} \mathrm{O}}-M_{\mathrm{CH}_{4}}$ - observed spectra.

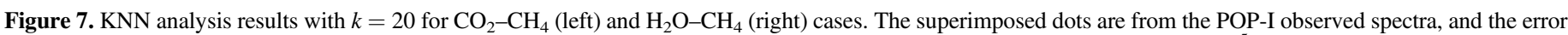

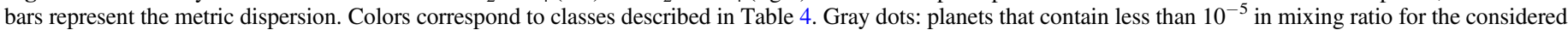

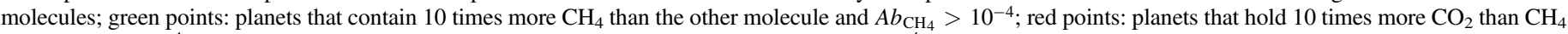

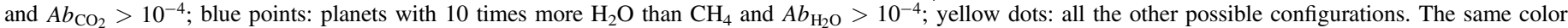

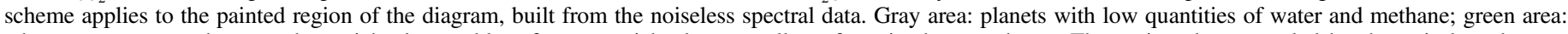

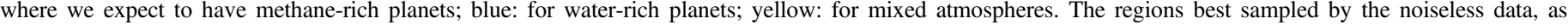
described in Section 2.4.1, are fully colored, while other regions are transparent.

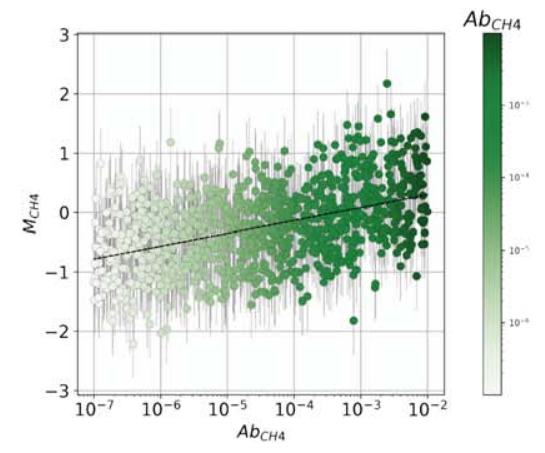

(a) $M_{\mathrm{CH}_{4}}$ versus $\mathrm{CH}_{4}$ true abundance value.

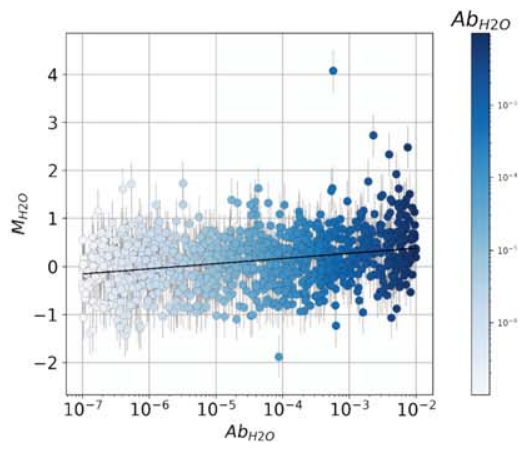

(b) $M_{\mathrm{H}_{2} \mathrm{O}}$ versus $\mathrm{H}_{2} \mathrm{O}$ true abundance value.

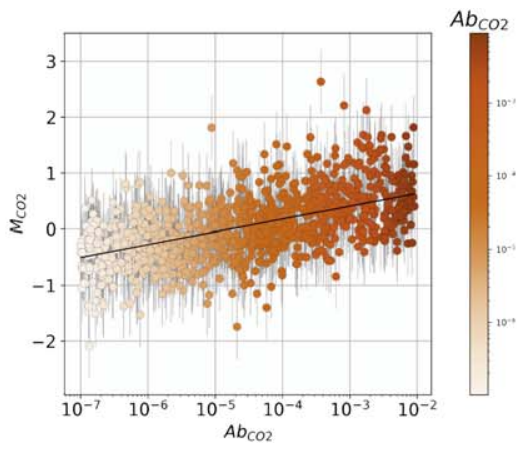

(c) $M_{\mathrm{CO}_{2}}$ versus $\mathrm{CO}_{2}$ true abundance value.

Figure 8. Comparison between the $M_{\text {mol }}$ estimates for each planet and the true molecular abundance value, $A b_{\text {mol }}$, in the atmospheres. $\mathrm{CH}_{4}, \mathrm{H}_{2} \mathrm{O}$, and $\mathrm{CO}_{2}$ cases are shown in the left, middle, and right panels, respectively. Data points with error bars represent POP-I planets. The color scale gives a visual representation of the molecular abundance in the atmosphere. A linear fit is shown by the solid black lines in each panel, with coefficients listed in Table 5. The fitted lines superimposed on the data highlight a positive correlation between the true molecular abundance values and the values estimated by the metric, $M_{\text {mol }}$.

We can use Figure 8 to obtain an estimate of the probability that a molecule mol has abundance in excess of $10^{-4}$, conditioned to the metric being larger than some value $M_{\mathrm{mol}, *}$, i.e., $P\left(A b_{\mathrm{mol}}>10^{-4} \mid M_{\mathrm{mol}}>M_{\mathrm{mol}, *}\right)$. For this, we can use the well-known chain rule for the conditional probability that states that $P(A \mid B)=P(A \cap B) / P(B)$, where $A$ and $B$ are two separate events. We estimate the number of data points found in a region of the diagrams of Figure 8 where both conditions are satisfied (favorable outcomes) divided by the number of data points for which only the condition $M_{\mathrm{mol}}>M_{\mathrm{mol}, *}$ is satisfied (total outcomes). From POP-I observed spectra, we can obtain a single realization of $P$. Therefore, we simulate 1000 realizations of POP-I observed spectra, using the same input noiseless POP-I population spectra and randomizing the noise realizations. In this way we simulate 1000 realizations of $P$ from which medians and $1 \sigma$ confidence levels are computed.

Figure 9 suggests that the metric can be used to classify planetary primary atmospheres for the presence of $\mathrm{CH}_{4}$ and $\mathrm{CO}_{2}$, and to a less extent $\mathrm{H}_{2} \mathrm{O}$, and atmospheres that are likely missing these molecular contributions. With reference to Figure 9, it can be seen that when $M_{\mathrm{CH}_{4}} \geqslant 0.5$, the number of planets wrongly classified to have $A b_{\mathrm{CH}_{4}}>10^{-4}$ is only $20 \%$, or 1 out of 5 are false positives. However, and as expected, the case of water is different, and our metric is not as effective in detecting the presence of water as it is for the other molecules. Even for large values of $M_{\mathrm{H}_{2} \mathrm{O}}$, the rate of false positives is close to $40 \%$. 


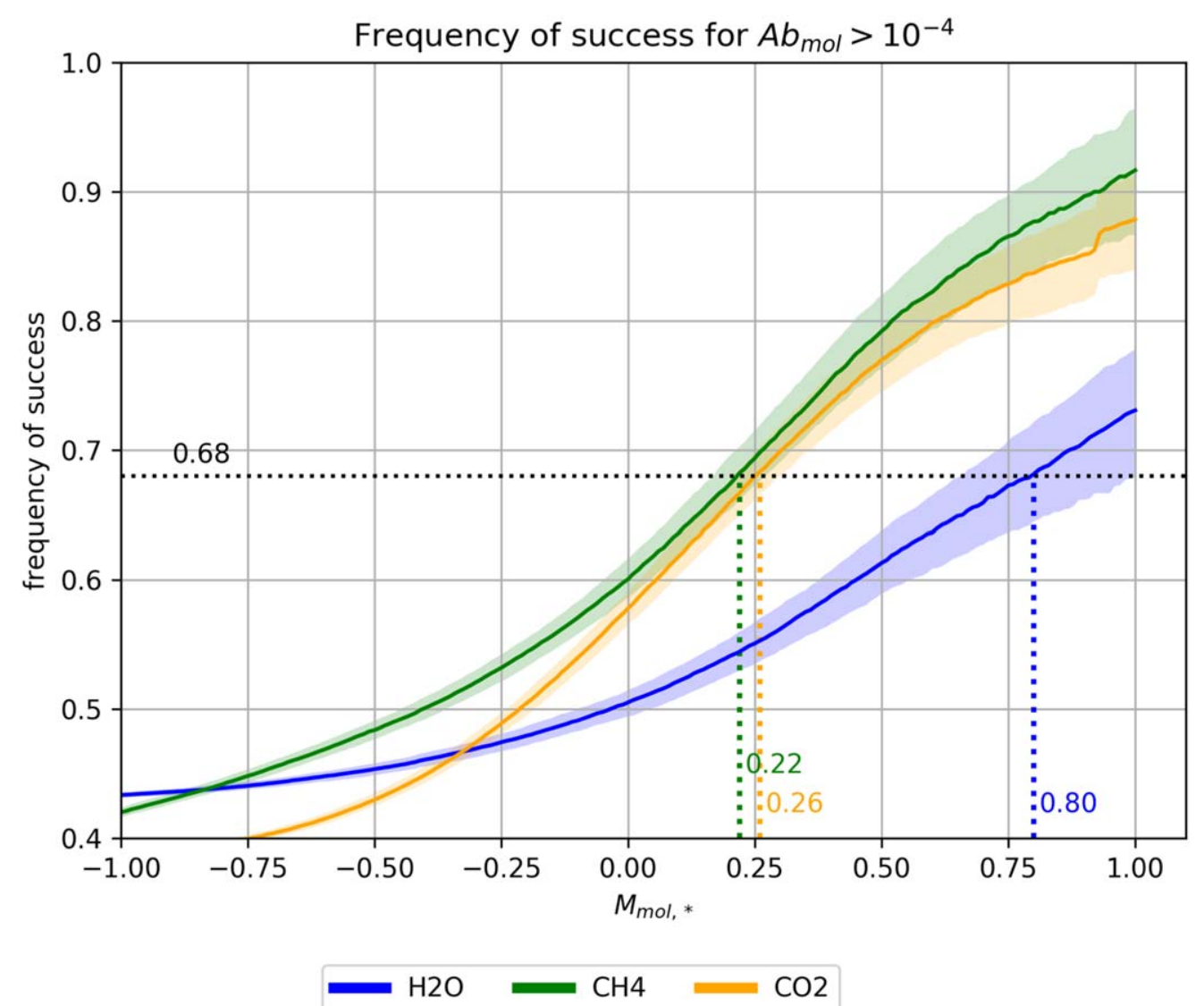

Figure 9. Probability that a molecule mol has abundance in excess of $10^{-4}$, conditioned to the metric being larger than some value $M_{\text {mol, } *}$, i.e., $P\left(A b_{\mathrm{mol}}>10^{-4} \mid\right.$ $\left.M_{\mathrm{mol}}>M_{\mathrm{mol}, *}\right) . \mathrm{CH}_{4}, \mathrm{H}_{2} \mathrm{O}$, and $\mathrm{CO}_{2}$ cases are shown by the green, blue, and orange lines, respectively. The lines are computed as the median of the probability estimates from 1000 different realizations of the POP-I observed population. The shaded regions are the $1 \sigma$ confidence levels associated with the median probability. Vertical dotted lines mark metric values, $M_{\mathrm{mol}, *}$, corresponding to a probability of $68 \%$.

Table 5

Fitted $C_{0}$ and $C_{1}$ Coefficients for $M_{\mathrm{mol}}=C_{0} \cdot \log \left(A b_{\mathrm{mol}}\right)+C_{1}$ for All the Possible Combinations of Considered Molecules

\begin{tabular}{lcccc}
\hline \hline & $\log \left(A b_{\mathrm{H}_{2} \mathrm{O}}\right)$ & $\log \left(A b_{\mathrm{CH}_{4}}\right)$ & $\log \left(\mathrm{Ab}_{\mathrm{CO}_{2}}\right)$ & $\log \left(\mathrm{Ab}_{\mathrm{NH}_{3}}\right)$ \\
\hline \multicolumn{5}{c}{$C_{0}$ Coefficients } \\
\hline$M_{\mathrm{H}_{2} \mathrm{O}}$ & 0.108 & -0.193 & -0.033 & 0.104 \\
& $( \pm 0.022)$ & $( \pm 0.022)$ & $( \pm 0.022)$ & $( \pm 0.022)$ \\
$M_{\mathrm{CH}_{4}}$ & 0.003 & 0.215 & -0.258 & 0.104 \\
& $( \pm 0.022)$ & $( \pm 0.022)$ & $( \pm 0.022)$ & $( \pm 0.022)$ \\
$M_{\mathrm{CO}_{2}}$ & -0.094 & -0.057 & 0.228 & -0.104 \\
& $( \pm 0.022)$ & $( \pm 0.022)$ & $( \pm 0.022)$ & $( \pm 0.022)$ \\
\hline \multicolumn{5}{c}{$C_{1}$ Coefficients } \\
& 0.599 & -0.756 & -0.028 \\
$M_{\mathrm{H}_{2} \mathrm{O}}$ & $( \pm 0.102)$ & $( \pm 0.106)$ & $( \pm 0.105)$ & $( \pm 0.105)$ \\
& -0.241 & 0.725 & -1.419 & 0.215 \\
$M_{\mathrm{CH}_{4}}$ & $( \pm 0.102)$ & $( \pm 0.106)$ & $( \pm 0.105)$ & $( \pm 0.105)$ \\
& -0.357 & -0.202 & 1.088 & -0.411 \\
$M_{\mathrm{CO}_{2}}$ & $( \pm 0.102)$ & $( \pm 0.106)$ & $( \pm 0.105)$ & $( \pm 0.105)$ \\
& &
\end{tabular}

Note. The bands used for $M_{\mathrm{mol}}$ are reported in Table 3.

\subsection{Deep and Machine Learning}

The percentages of correct classifications for all considered molecules and for different minimum input abundances are reported in Table 6(a) for KNN, in Table 6(b) for MLP, in Table 6(c) for RFC, and in Table 6(d) for SVC.
Table 6 shows that for all deep-learning and ML algorithms, the percentages of success in identifying the presence of molecules inside the atmosphere grow with the minimum molecular abundances that we set as a threshold for the classification. While this is expected, it may come as a surprise that in general these algorithms appear to be effective in detecting the presence of all individual molecules with a relatively small fraction of false positives (about $30 \%$ or smaller) even at low abundances. This is perhaps because ML algorithms learn to classify atmospheres by recognizing spectral shapes. These algorithm performances can be to a certain level independent of the molecules considered, as long as the training set contains sufficiently diverse spectra to allow a secure identification, including water in the presence of ammonia or biases, which is where our metric shows its more severe weaknesses. We also notice from Table 6 that KNN, MLP, RFC, and SVC show comparable overall performance, and that $\mathrm{CH}_{4}$ and $\mathrm{CO}_{2}$ are the most straightforward molecules to identify in Tier 1 planetary spectra.

A comparison between these results and our metric is presented in Section 4.5.

\section{Discussion}

In this section, we discuss the metric results shown in Section 3.2. We first discuss the bias (Section 4.1), and then we focus on the metric characteristics, such as the relation between the metric estimates and the input molecular abundances (Section 4.2), and the detection limits (Section 4.3). Then, we compare the metric 


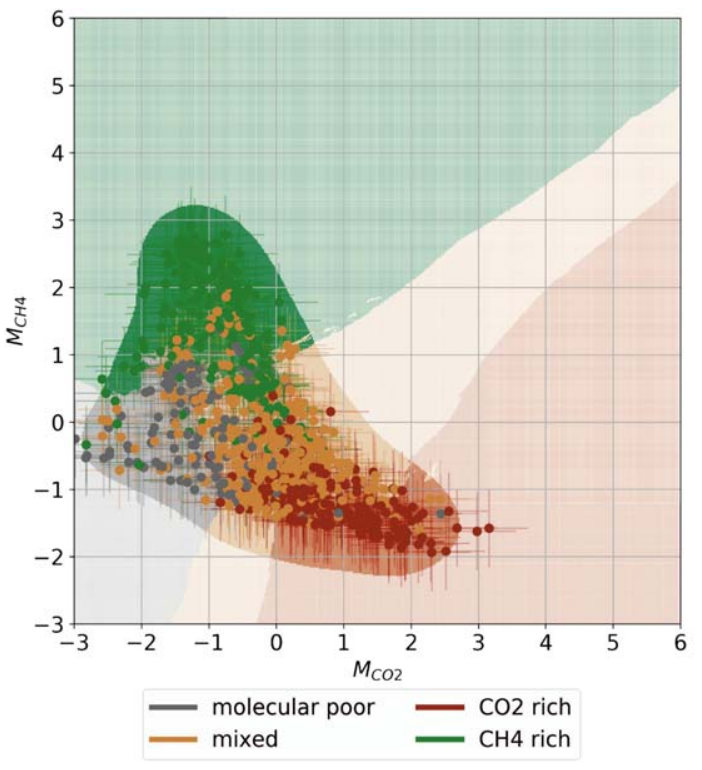

(a) $\mathrm{M}_{\mathrm{CO}_{2}}-\mathrm{M}_{\mathrm{CH}_{4}}$ - noiseless spectra.

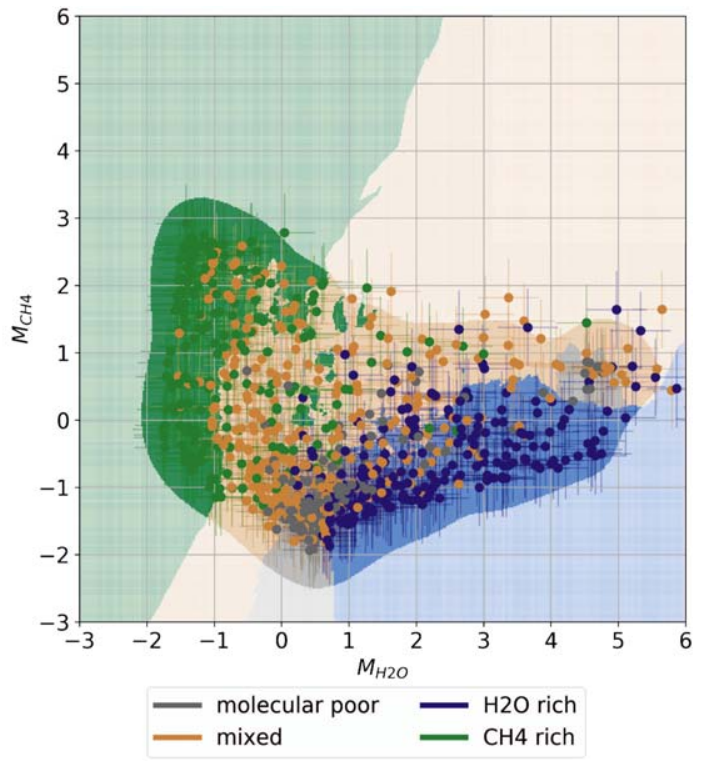

(b) $\mathrm{M}_{\mathrm{H}_{2} \mathrm{O}}-\mathrm{M}_{\mathrm{CH}_{4}}$ - noiseless spectra.

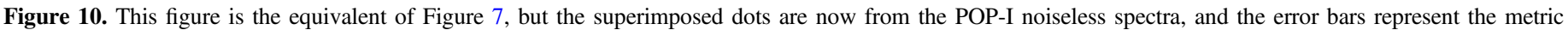

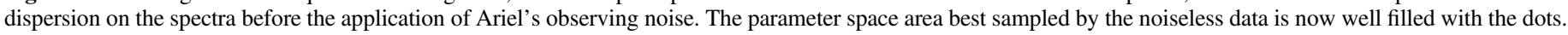

performance with a spectral retrieval (Section 4.4) and with deeplearning and ML algorithms (Section 4.5).

\subsection{Metric Bias}

The KNN analysis discussed earlier and shown in Figure 7 is trained on POP-I noiseless spectra, and the data points shown in that figure are obtained estimating the metric on POP-I observed spectra, as described in Section 2.4.1. To verify whether the metric is biased, the $\mathrm{KNN}$ analysis is repeated with data points obtained estimating the metric on POP-I noiseless spectra. This is shown in Figure 10, which should be compared with Figure 7. The background colors are very similar in both cases, with small variations due to the training process that selects randomly $70 \%$ POP-I noiseless examples. In absence of biases, we expect the distribution of observed data points to be that of noiseless data points, convolved with the distribution of the noise. However, it can be noticed from the comparison of the two figures that the distribution of the observations is more clustered toward the origin of the coordinate axes, compared to noiseless data points. This is a consequence of the bias introduced by the metric normalization discussed in Section 2.4: normalization is required such that the metric response is insensitive to the atmospheric scale height, and sensitive only to the presence of molecular signatures, at the cost of biasing the estimator. We should additionally point out that Figure 9 results are also affected by the bias. The observing noise reduces the $M_{\mathrm{mol}}$ average estimates, and therefore for smaller observing noise, the three colored lines in the figure are shifted to the right, and the $68 \%$ of success corresponds to higher $M_{\mathrm{mol}}$ values.

The work presented here demonstrates that the metric we have designed is a powerful tool capable of revealing the presence of a molecule in an atmosphere and that the prediction is independent of the type of the planet and its basic parameters (such as temperature, radius, and pressure) within the limits explored here. However, this comes at the cost of biasing the estimator by a quantity that depends on the instrumental noise as discussed in Section 2.4. Provided that the metric can be debiased, it can be used in a predictive way where
Table 6

Percentages of Correct Identifications for the Considered Molecules and with Different Thresholds

\begin{tabular}{lccc}
\hline \hline Molecule & $A b_{\text {mol }}>10^{-5}[\%]$ & $A b_{\text {mol }}>10^{-4}[\%]$ & $A b_{\text {mol }}>10^{-3}[\%]$ \\
\hline (a) KNN Percentages of Success to Identify Spectra Bearing Different Minimum Amounts \\
of Molecules \\
$\mathrm{CH}_{4}$ & 79 & 83 & 85 \\
$\mathrm{CO}_{2}$ & 77 & 79 & 82 \\
$\mathrm{H}_{2} \mathrm{O}$ & 64 & 71 & 82 \\
$\mathrm{NH}_{3}$ & 75 & 82 & 84 \\
\hline
\end{tabular}

(b) MLP Percentages of Success to Identify Spectra Bearing Different Minimum Amounts of Molecules

\begin{tabular}{llll}
\hline $\mathrm{CH}_{4}$ & 78 & 85 & 87 \\
$\mathrm{CO}_{2}$ & 77 & 81 & 83 \\
$\mathrm{H}_{2} \mathrm{O}$ & 70 & 76 & 84 \\
$\mathrm{NH}_{3}$ & 80 & 86 & 87 \\
\hline
\end{tabular}

(c) RFC Percentages of Success to Identify Spectra Bearing Different Minimum Amounts of Molecules

\begin{tabular}{llll}
\hline $\mathrm{CH}_{4}$ & 77 & 82 & 87 \\
$\mathrm{CO}_{2}$ & 76 & 79 & 83 \\
$\mathrm{H}_{2} \mathrm{O}$ & 69 & 74 & 82 \\
$\mathrm{NH}_{3}$ & 78 & 85 & 87 \\
\hline
\end{tabular}

(d) SVC Percentages of Success to Identify Spectra Bearing Different Minimum Amounts of Molecules

\begin{tabular}{llll}
\hline $\mathrm{CH}_{4}$ & 79 & 86 & 89 \\
$\mathrm{CO}_{2}$ & 79 & 83 & 84 \\
$\mathrm{H}_{2} \mathrm{O}$ & 69 & 78 & 84 \\
$\mathrm{NH}_{3}$ & 81 & 87 & 87 \\
\hline
\end{tabular}

Note. In each column we report a different minimum $A b_{\text {mol }}$, and in each row we report a different molecule. The percentages represent how many of the atmospheres have been correctly identified by the algorithm to have at least the specified minimum amount of that molecule, and therefore they represent the algorithm accuracy. Each ML algorithm has been trained on POP-III and tested on POP-I.

an observation (along with its dispersion estimate) can be compared to the calibrated (trained) metric space to infer the possible molecular content of the target. Because instrumental noise can be 


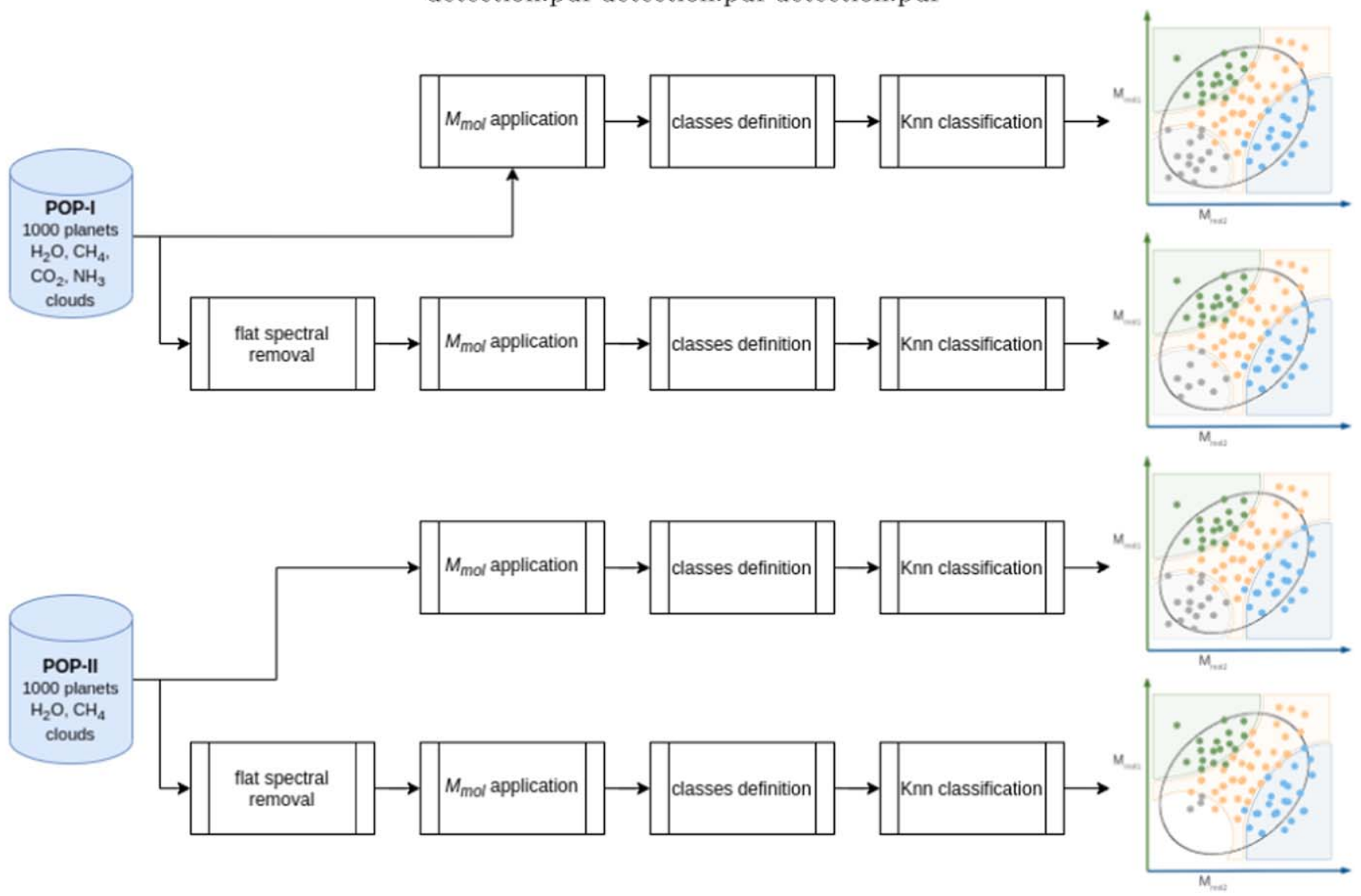

Figure 11. The figure shows the strategy adopted to identify the molecular detection limit for the developed metric. Starting from POP-I, we classify the planets as described in Section 2.4.1. Without removing the flat spectra from the population, we would end up with the same results described in Figure 4; by contrast, if we remove flat spectra, we end up with similar results but with fewer molecular-poor planets, because even without flat-spectrum atmospheres there will be planets bearing molecules different from the couple investigated by the plot. Different is the case of POP-II: here we have only two molecules in the population, and therefore if we remove the flat-spectrum planets, we will end with no molecular-poor atmospheres.

well characterized, it would be possible to debias the metric estimator. This requires a detailed noise analysis, taking into account the uncertainties on the noise estimates, which is beyond the scope of this paper. In the rest of this section we focus on what we can learn from this kind of analysis provided that the metric can be debiased, and we leave to future work a detailed study on how this debiasing can be secured.

\subsection{Relation with the Input Abundances}

We see in Figure 8 that the correlation between $M_{\mathrm{mol}}$ and $\log \left(A b_{\mathrm{mol}}\right)$ is in general not strong enough to quantify the input molecular abundances. This is because atmospheric spectra are made of complex nonlinear contributions from all the molecules. Therefore, a method based only on spectral shapes (i.e., this metric) is inadequate to quantify molecular abundances. However, the goal of this metric, provided that the bias can be removed, is not to assess the abundance of a certain species in the planet atmosphere, but only its possible presence, avoiding the use of spectral retrieval techniques, which may not be indicated for Tier 1 data.

Focusing on Table 5 and looking at the coefficients fitted for $M_{\mathrm{H}_{2} \mathrm{O}}$ over $\log \left(A b_{\mathrm{H}_{2} \mathrm{O}}\right)$ and over $\log \left(A b_{\mathrm{NH}_{3}}\right)$, we may infer that the metric may not be effective to distinguish between water and ammonia. However, the degeneracy can be broken by performing a spectral retrieval if the target was observed at Ariel Tier $2 \mathrm{~S} / \mathrm{N}$, as shown in an example in Section 4.4. This population analysis is based on the study of spectral shapes only, and it does not make use of parameters such as planetary mass, radius, and temperature. Although it has proven difficult to distinguish between water and ammonia with this metric, using some knowledge of planetary properties may help us to disentangle the two molecules in a future work; for example, while a Neptune can hold ammonia, a hot Jupiter planet is not expected to. One of the goals of Tier 1 is to identify targets with interesting spectra to be reobserved in higher$\mathrm{S} / \mathrm{N}$ tiers. From this point of view, even if the metric cannot clearly separate between water and ammonia, it can suggest the presence of interesting molecules in the spectrum. This can in turn be used to make informed decisions about targets to be selected for further studies.

\subsection{Metric Detection Limit}

To explore the detection limit of molecules by the metric, we examine the molecular-poor/spectral flat region of Figure 2. A planet spectrum would be found in that region because of (i) clouds, (ii) a low temperature (i.e., small scale height), (iii) low molecular abundances, or a combination of the three. In all cases, the spectrum is expected to be featureless, i.e., flat. Point (iii) is defined from input abundances smaller than $10^{-5}$ (Table 4). The metric detection limit can then be investigated by removing flat spectra before training the $\mathrm{KNN}$, by 


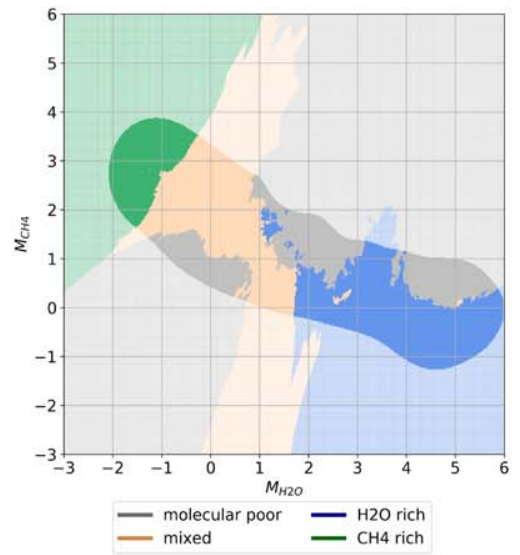

(a) KNN classification map for $\mathrm{CH}_{4}$ and $\mathrm{H}_{2} \mathrm{O}$, including flat spectra. Molecular poor planets defined as $A b_{C_{4}}$ and $A b_{H_{2} O}<$ $10^{-5}$.

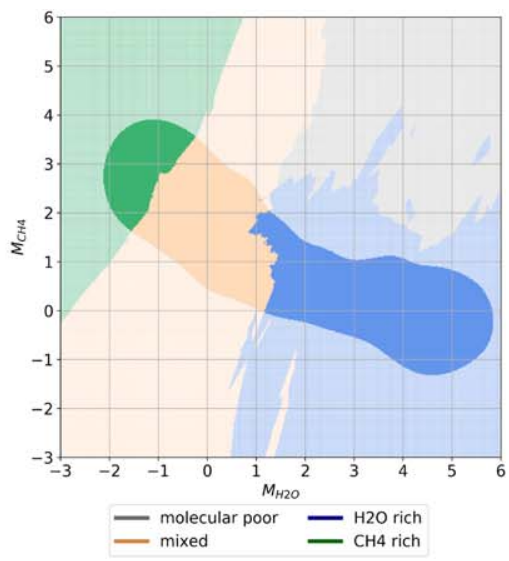

(b) KNN classification map for $\mathrm{CH}_{4}$ and $\mathrm{H}_{2} \mathrm{O}$ without flat spectra. Molecular poor planets defined as $A b_{\mathrm{CH}_{4}}$ and $A b_{\mathrm{H}_{2} \mathrm{O}}<$ $10^{-5}$.

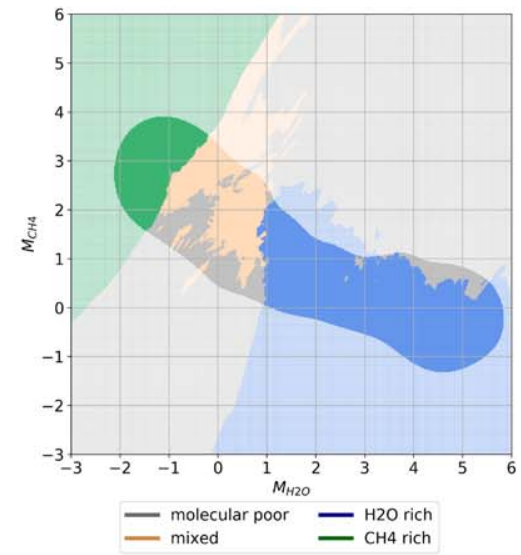

(c) KNN classification map for $\mathrm{CH}_{4}$ and $\mathrm{H}_{2} \mathrm{O}$ without flat spectra. Molecular poor planets defined as $A b_{\mathrm{CH}_{4}}$ and $A b_{\mathrm{H}_{2} \mathrm{O}}<$ $10^{-4}$.

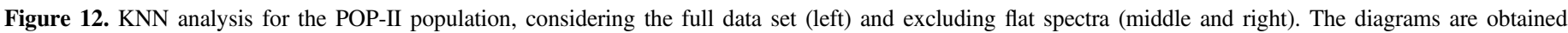
following the bottom branches of Figure 4: we used the noiseless planetary spectra to classify the metric space and to select the best-sampled regions.

increasing before training the molecular-poor spectra threshold to above $10^{-5}$, and by monitoring the KNN classification results. As the threshold increases, we expect the $\mathrm{KNN}$ to begin failing the molecular-poor/flat classification when spectra can no longer be considered flat.

We perform the KNN training on the noiseless spectra of both POP-I and POP-II, the latter containing only $\mathrm{CH}_{4}$ and $\mathrm{H}_{2} \mathrm{O}$, the former containing all molecules considered in this work. Each noiseless spectrum has its associated observed spectrum. Flat spectra are identified on observed spectra, and the corresponding noiseless spectra are ignored in the KNN training.

The motivation behind using POP-II is as follows. If we have a population containing only $\mathrm{CH}_{4}$ and $\mathrm{H}_{2} \mathrm{O}$ and we properly remove all planets with a flat spectrum, there should be no targets left with nondetectable molecular features. In the case of POP-I, however, we do not expect all the planets with $A b_{\mathrm{CH}_{4}}$ and $A b_{\mathrm{H}_{2} \mathrm{O}}<10^{-5}$ to be flat, because other molecules $\left(\mathrm{CO}_{2}\right.$ and $\left.\mathrm{NH}_{3}\right)$ can show features. Therefore, the flat-spectrum removal procedure will not empty the molecular-poor planet class in this population. Using POP-II instead, we expect that, after removing all flat planets, there will not be molecular-poor atmospheres anymore. The procedure is summarized in Figure 11.

The outcome of this analysis is shown for POP-I and POP-II in Figures 12 and 13, respectively. Only the calibrated regions are shown, and data points have been omitted for clarity. Figure 12(a) shows the POP-II KNN analysis with all planets and planetary classes of Table 4, in Figure 12(b) the KNN is trained removing flat spectra from the training set, and in Figure 12(c) the training is done removing flat spectra first, and increasing the threshold of molecular-poor spectra from $A b_{\mathrm{mol}}<10^{-5}$ to $A b_{\mathrm{mol}}<10^{-4}$. We notice that Figure 12(b) shows no molecular-poor atmosphere after excluding spectrally flat cases. This confirms that our metric is able to separate the more complex atmospheres from the flat ones in the simple case of only two molecules. By contrast, Figure 12(c) still shows a gray area, signifying that atmospheres with $10^{-5}<$ $A b_{\text {mol }}<10^{-4}$ cannot be considered flat. This can be interpreted as a molecular detection limit. We also notice from the figure that these spectra populate the lower left corner of the best-sampled area of the diagram, meaning that they are classified as having the smallest spectral features of the samples. This confirms the relation between the metric and the molecule abundance. The detection limit is expected to improve in Tier 2 observations, and Changeat et al. (2020) find that the detection limit using spectral retrieval techniques on Tier 2 is about two orders of magnitude smaller compared to that of the metric.

In Figure 13 we remove all flat spectra from the planetary population POP-I and report the results of KNN analysis. Here we see that, as expected, while removing all flat spectra from POP-II does also remove all molecular-poor instances, the same does not occur in POP-I. In this case, molecular-poor spectra in any two molecules, such as $\mathrm{CH}_{4}-\mathrm{CO}_{2}$ or $\mathrm{CH}_{4}-\mathrm{H}_{2} \mathrm{O}$, may appear nonflat because of the presence of the other two molecules, i.e., $\mathrm{NH}_{3}-\mathrm{H}_{2} \mathrm{O}$ or $\mathrm{NH}_{3}-\mathrm{CO}_{2}$, respectively.

\subsection{Input Abundance Retrieval}

We compare here two atmospheric retrievals of the same planet observed both in Tier 1 and in Tier 2. This exercise has two goals:

1. to confirm that a spectral retrieval is capable of disentangling water and ammonia, and to constrain the atmospheric composition of POP-I targets observed in Tier 2 with Ariel; and

2. to show that even though it is possible to perform a spectral retrieval on Tier 1 data for some selected planets, its performance is comparable to that of the metric.

From the POP-I planets, we select one that has water and ammonia in high abundances, low cloud presence, high temperature, and a diameter larger than Jupiter's. Such selection will help us to investigate the capability of Tier 2 observed data (simulated as described in Section 2.2) to break the water-ammonia degeneracy, as well as to estimate the uncertainties from a retrieval using Tier 1 observed data only. 


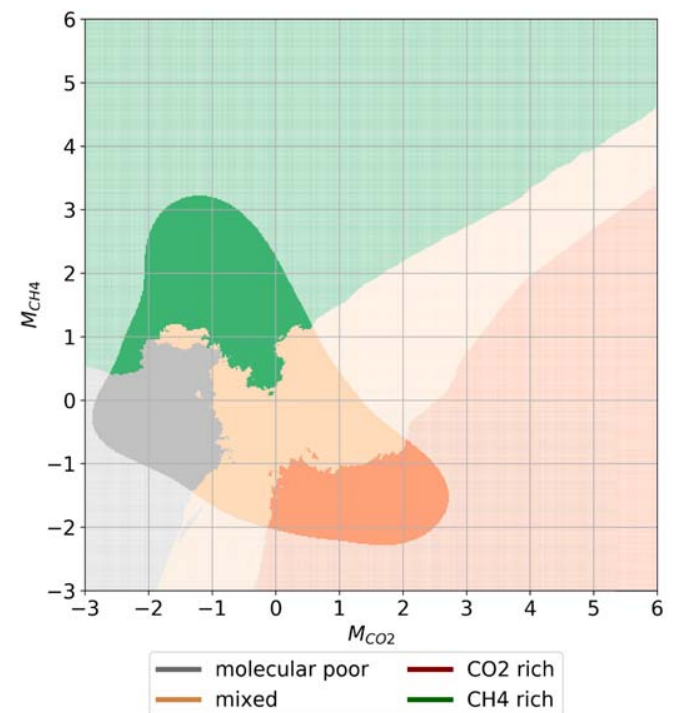

(a) $\mathrm{KN}$ neighbours for POP-I population without flat spectra. $\mathrm{CH}_{4}-\mathrm{CO}_{2}$ case.

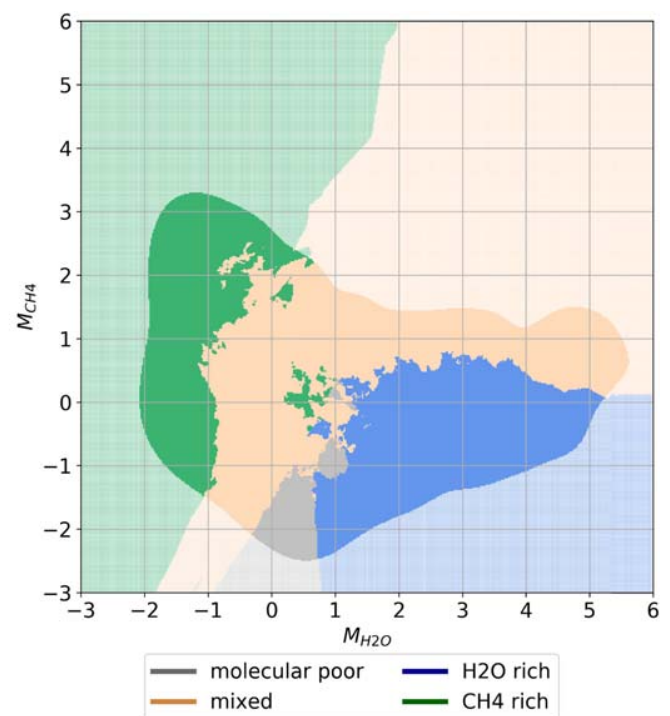

(b) KN neighbours for POP-I population without flat spectra. $\mathrm{CH}_{4}-\mathrm{H}_{2} \mathrm{O}$ case.

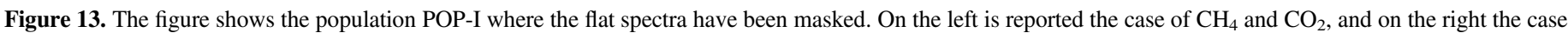

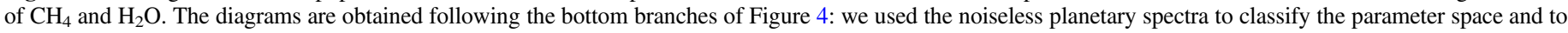

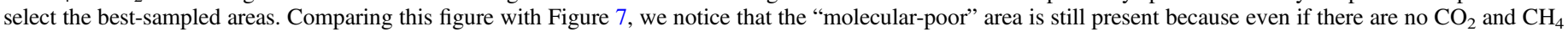
in the planet atmosphere, there could be $\mathrm{NH}_{3}$ and $\mathrm{H}_{2} \mathrm{O}$ having features (left case), or if there are no $\mathrm{H}_{2} \mathrm{O}$ and $\mathrm{CO}_{2}$, there could be $\mathrm{NH}_{3}$ and $\mathrm{CO}_{2}$ (right case).

Table 7

Retrieval Parameter Table Showing Fit Boundaries, True Inputs, and Retrieved Parameters with Uncertainties for Tier 1 and Tier 2 Observations

\begin{tabular}{ccccc}
\hline \hline Name & Boundaries & $\begin{array}{c}\text { True } \\
\text { Value }\end{array}$ & $\begin{array}{c}\text { Tier 1 } \\
\text { Retrieved }\end{array}$ & $\begin{array}{c}\text { Tier 2 } \\
\text { Retrieved }\end{array}$ \\
\hline$R_{p}\left[R_{\text {Jup }}\right]$ & {$[0.5 \rightarrow 2]$} & 1.24 & $1.241_{-0.004}^{+0.004}$ & $1.2412_{-0.0011}^{+0.0020}$ \\
$T_{p}[K]$ & {$[800 \rightarrow 2400]$} & 1617 & $1720_{-133}^{+153}$ & $1693_{-38}^{+42}$ \\
$\log \left(\mathrm{CH}_{4}\right)$ & {$[-8 \rightarrow-2]$} & -3.13 & $-4.11_{-2.55}^{+1.51}$ & $-3.08_{-0.18}^{+0.23}$ \\
$\log \left(\mathrm{CO}_{2}\right)$ & {$[-8 \rightarrow-2]$} & -3.44 & $-3.74_{-1.61}^{+0.93}$ & $-3.59_{-0.26}^{+0.30}$ \\
$\log \left(\mathrm{H}_{2} \mathrm{O}\right)$ & {$[-8 \rightarrow-2]$} & -2.93 & $-2.63_{-0.85}^{+0.42}$ & $-2.96_{-0.17}^{+0.20}$ \\
$\log \left(\mathrm{NH}_{3}\right)$ & {$[-8 \rightarrow-2]$} & -2.91 & $-2.73_{-0.73}^{+0.43}$ & $-3.03_{-0.15}^{+0.22}$ \\
$\log \left(P_{\text {clouds }}\right)$ & {$[-3 \rightarrow 6]$} & 5.90 & $4.76_{-0.83}^{+0.81}$ & $4.89_{-0.74}^{+0.75}$ \\
\hline
\end{tabular}

Note. As in Figure 14, the notation $\log (X)$, where $X$ is one of $\mathrm{CH}_{4}, \mathrm{CO}_{2}, \mathrm{H}_{2} \mathrm{O}$, or $\mathrm{NH}_{3}$, represents the retrieved logarithm of the molecular abundance of the given species and should be compared to the input $\log \left(A b_{X}\right)$.

To perform the retrieval, we use TauREx 3 (Al-Refaie et al. 2021). The parameters fitted with fit boundaries, true values, and retrieved values are listed in Table 7, while the retrieved solutions and posteriors are shown in Figure 14.

For the selected planet, we notice that in Tier 2 the abundances of the molecules considered are well constrained, and, as expected, low-level (high-pressure) clouds are undetected in both cases.

The Tier 1 results can be linked to our previous analysis on molecular input abundance detection (Section 4.2). We compute the probability to have molecular abundances greater than $10^{-4}$ from the retrieval posteriors and compare these with the probability obtained with our metric (Figure 9). In this case, the measured $M_{\mathrm{mol}}$ are $M_{\mathrm{CH}_{4}}=-0.47, M_{\mathrm{CO}_{2}}=0.54$, and $M_{\mathrm{H}_{2} \mathrm{O}}=0.29$. The results are listed in Table 8. Tier 2 observations provide a confident detection of methane, carbon dioxide, and water, while Tier 1 retrievals are broadly comparable to our metric approach in detecting the presence of these molecules.

These results appear to confirm that spectral retrievals may not be best suited or at the very least necessary to analyze Tier 1 data. Retrievals are model dependent, and one needs to define planet parameters, as well as cross sections, pressure-temperature profiles, etc. Priors might need to be imposed to ensure convergence. Retrievals are also computationally expensive, making it not trivial to conduct the analysis on hundreds of targets. A photometric metric, instead, is model independent, which may be an advantage when assessing a planet observation for the first time. The full analysis takes only minutes on a desktop computer to reduce 1000 observations.

\subsection{Comparison with Deep and Machine Learning}

ML techniques are difficult to interpret, and so a comparison between their performance and that of our metric can help us in gaining confidence in the outcomes from ML classifiers. For this purpose, we consider a planet as bearing a molecule if $A b_{\text {mol }}>10^{-4}$. Then, with our metric we select all planets that have $M_{\mathrm{CH}_{4}} \geqslant 0.22$, which, according to Figure 9, corresponds to a probability of $\sim 68.3 \%$ to have an $A b_{\text {mol }}>10^{-4}$ for $\mathrm{CH}_{4}$. We repeat the same procedure, letting $M_{\mathrm{CO}_{2}} \geqslant 0.26$ for $\mathrm{CO}_{2}$ and $M_{\mathrm{H}_{2} \mathrm{O}} \geqslant 0.80$ for $\mathrm{H}_{2} \mathrm{O}$. In each sample, we check how many of the selected planets have molecular abundances in excess of $10^{-4}$, obtaining a percentage of success for our metric (or metric precision). In the same way, we check how many of the planets flagged by each of the deep-learning and ML algorithms in the full sample actually bear the molecules, such that we can compare their precision performance in Table 9.

We notice a marginally better success rate for deep-learning and ML algorithms in the cases of KNN and MLP, while RFC and SVC algorithms suggest a better performance when compared to that of the metric. Better performances are expected because, while our metric considers only specific bins 

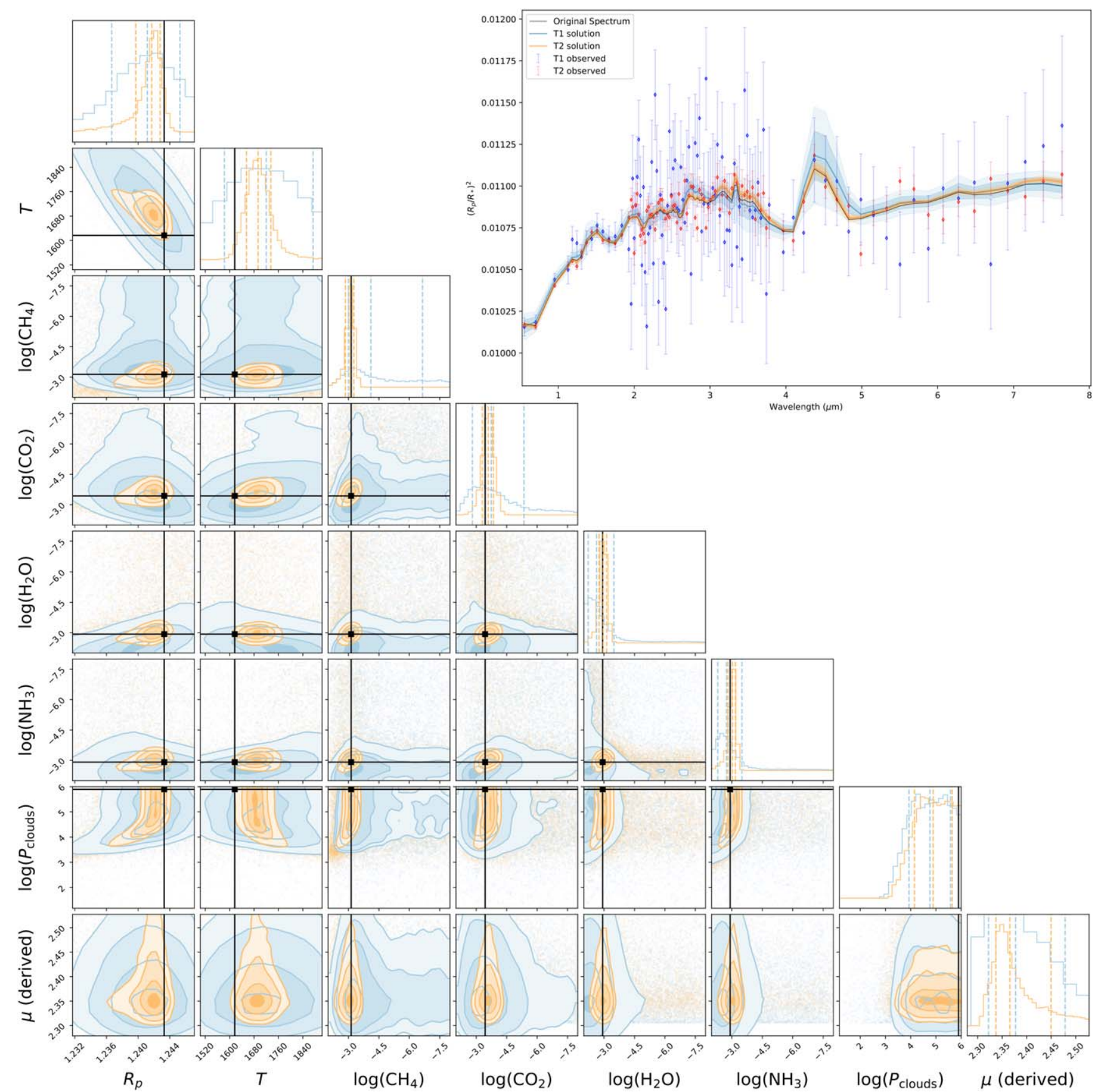

Figure 14. Retrieved spectra and posteriors. The corner plot shows the posteriors for each retrieved parameter using Tier 1 (blue) and Tier 2 (orange) observed data. Input values are shown by the black lines. The top right panel shows the retrieved spectra from Tier 1 (blue) and Tier 2 data using colored shaded bands for $1 \sigma$ and $2 \sigma$ uncertainties and the input (black solid line). The notation $\log (X)$, where $X$ is one of $\mathrm{CH}_{4}, \mathrm{CO}_{2}, \mathrm{H}_{2} \mathrm{O}$, or $\mathrm{NH}_{3}$, represents the logarithm of the molecular abundance of the given species and should be compared to $\log \left(A b_{X}\right)$.

Table 8

Probability to Have $A b_{\mathrm{mol}}>10^{-4}$ for Each Molecule Computed from $M_{\mathrm{mol}}$ and from Tier 1 and Tier 2 Retrieval Posteriors

\begin{tabular}{lccc}
\hline \hline Molecule & $M_{\text {mol }}[\%]$ & Tier 1 [\%] & Tier 2 [\%] \\
\hline $\mathrm{CH}_{4}$ & 49 & 48 & 100 \\
$\mathrm{CO}_{2}$ & 78 & 58 & 94 \\
$\mathrm{H}_{2} \mathrm{O}$ & 56 & 89 & 100 \\
\hline
\end{tabular}

Note. The numbers refer to the planet case discussed in Section 4.4. in the spectrum, the classification algorithms gather information from all the spectral data points. The comparable performance of the metric with the KNN and MLP suggests that the molecular bands chosen for the metric are not far from ideal, but the comparatively better performances of RFC and SVC provide an indication that margins for improvement may exist.

While more work is required along this path, which is beyond the scope of this work, deep learning and ML appear to 
Table 9

Percentages of Positive Detection for Our Metric, Compared to Deep-learning Algorithm Precision.

\begin{tabular}{lccccc}
\hline \hline Molecule & $M_{\text {mol }}[\%]$ & KNN [\%] & MLP[\%] & RFC[\%] & SVC[\%] \\
\hline $\mathrm{CH}_{4}$ & $69^{\mathrm{a}}$ & 75.4 & 84.2 & 92.5 & 90.1 \\
$\mathrm{CO}_{2}$ & 68.3 & 71.4 & 75.8 & 83.1 & 83.5 \\
$\mathrm{H}_{2} \mathrm{O}$ & 68.3 & 74.5 & 79.0 & 96.7 & 99.4 \\
\hline
\end{tabular}

Notes. To assess the presence of a molecule, we flag a planet if $A b_{\mathrm{mol}}>10^{-4}$. We investigate $\mathrm{CH}_{4}$ in the first row, $\mathrm{CO}_{2}$ in the second, and $\mathrm{H}_{2} \mathrm{O}$ in the third, selecting the planets with $M_{\mathrm{CH}_{4}} \geqslant 0.22$ (first row), $M_{\mathrm{CO}_{2}} \geqslant 0.26$ (second row), and $M_{\mathrm{H}_{2} \mathrm{O}} \geqslant 0.80$ (third row).

${ }^{a}$ These percentages arise from a discrete distribution of data, and therefore we cannot exactly identify the $68.3 \%$ quantity. In this case $69 \%$ is the closest possible value.

be very promising for this classification problem, and we shall leave to dedicated works, such as the one presented in Hou Yip et al. (2021), a more exhaustive investigation of these techniques, their comparison with more physically motivated strategies similar to the metric, and a thorough investigation of biases that may affect all these techniques.

\section{Conclusion}

This work presents data analysis methods to extract atmospheric information from Ariel Tier 1 observations of a large and diverse sample of exoplanets. Ariel's Tier 1 has been optimized as a reconnaissance survey of exoplanets, with $\mathrm{S} / \mathrm{N}$ larger than 7 after averaging the observed spectra in about seven photometric data points over the $0.5-7.8 \mu \mathrm{m}$ wavelength range. Therefore, having only seven effective data points per spectrum, Tier 1 data may not be ideally suited for detailed spectral retrieval and to constrain chemical abundances, for which Tier 2 or Tier 3 observations are needed. However, Tier 1 data contain a wealth of information, such as the spectral signatures of important molecules, whose presence can in principle be detected, therefore enabling targets to be classified, and can be used to assess planets with featureless spectra.

In this work we simulate the entire population of exoplanets using Alfnoor, assigning a randomized atmosphere to each planet in the Ariel Mission Reference Sample, which comprises a diverse population of 1000 exoplanetary targets. We consider primary atmospheres with contributions from clouds, methane, water, carbon dioxide, and ammonia. This simulated data set is expected to be representative of the Ariel Tier 1 reconnaissance survey.

The aim of this paper is threefold: (1) to show the capability of Tier 1 to detect featureless spectra, (2) to define a metric to classify and select planets to be reobserved in higher-resolution tiers, and (3) to introduce other strategies that can be used to maximize the science exploitation of Ariel's Tier 1 data, for consideration in future studies.

(1) We presented a reliable method to identify flat spectra. By dividing the Ariel wavelength range into four bands, we classify as flat those planets where the four spectral bands' response is compatible with a flat line, following a $\chi^{2}$ test.

(2) We developed a model-independent metric that bins the observed spectra over selected bands bearing the signatures of the molecules under investigation. From the observed spectrum alone, this method proves capable of indicating the presence of an atmosphere and its possible composition, independently of planet parameters such as mass, size, and temperature. Applying the metric to a Tier 1 observed spectrum, we find a $1 \sigma$ confidence level in identifying $\mathrm{CH}_{4}$, $\mathrm{CO}_{2}$, or $\mathrm{H}_{2} \mathrm{O}$ when their abundance in the atmosphere is in excess of $10^{-4}$ in mixing ratio, and their estimates $M_{\mathrm{CH}_{4}} \geqslant 0.22, \quad M_{\mathrm{CO}_{2}} \geqslant 0.26$, or $M_{\mathrm{H}_{2} \mathrm{O}} \geqslant 0.80$, respectively, demonstrating how the metric may be used in a statistically quantitative way. However, we find that the metric is biased, and the bias depends on the magnitude of the instrumental noise. Debiasing the metric is required for its predictions to be quantitative. Debiasing is expected to be possible, following a detailed characterization of the instrumental uncertainties, and we reserve an investigation of these aspects for a future study. The metric struggles to separate $\mathrm{H}_{2} \mathrm{O}$ and $\mathrm{NH}_{3}$. This may be partially due to the effect of a bias, or, more likely, because of the two molecules partially overlapping features. However, the metric is successful in classifying these targets as having an atmosphere. Should these targets be selected for Tier 2 observations, a spectrum retrieval analysis can constrain all abundances to high significance.

(3) We have performed a preliminary comparison of four different deep-learning and ML algorithms for the chemical classification of Tier 1 atmospheres. We find that their performance in identifying the presence of a certain molecule in the spectrum is marginally better than that of the metric in the case of KNN and MLP, but RFC and SVC outperform the metric, justifying a detailed follow-up study in future work.

This work has been supported by ASI grant No. 2018.22.HH.0, ASI grant No. 2021.5.HH.0, and the UCL Cities Partnerships Programme. The project also received funding from the European Research Council (ERC) under the European Union's Horizon 2020 research and innovation program (grant agreement No. 758892, ExoAI), and under the European Union's Seventh Framework Programme (FP7/2007-2013)/ERC grant agreement No. 617119 (ExoLights). Furthermore, we acknowledge funding by the Science and Technology Funding Council (STFC) grants ST/K502406/1, ST/P000282/1, ST/P002153/1, ST/S002634/ 1 , and by the UK Space Agency grant ST/W00254X/1. The authors acknowledge the contribution of the anonymous referees that greatly improved this work.

\section{Appendix \\ Analytical Derivation of the Metric}

As mentioned in Section 2.4, the metric here presented is (i) sensitive to the molecules, (ii) independent of the planet size, and (iii) independent of the scale height. To show it, we start by using the following notation: in transmission spectroscopy we are measuring

$$
\frac{\Delta f}{f}(\lambda)=\frac{R_{\mathrm{pl}}^{2}+2 R_{\mathrm{pl}} \cdot z(\lambda)}{R_{\star}^{2}},
$$

where $f$ is the measured flux from the star, $\Delta f$ is the difference between the flux measured during the transit and the one measured out of transit, $R_{\rho}$ and $R_{\star}$ are the planet and the star radii, respectively, and $z(\lambda)$ is the measured wavelengthdependent transit depth. Now, applying the definition of $z(\lambda)$ 
from Lecavelier Des Etangs et al. (2008),

$$
z(\lambda)=H \ln \left(\frac{\epsilon_{\mathrm{abs}} \sigma_{\mathrm{abs}}(\lambda) P_{0}}{\tau_{\mathrm{eq}}} \sqrt{\frac{2 \pi R_{p} H}{k_{B}^{2} T_{p}^{2}}}\right),
$$

where $\epsilon_{\mathrm{abs}}$ and $\sigma_{\mathrm{abs}}$ are the abundance and cross section of the main absorbent at the $\lambda$ wavelength. $H$ is the scale height to which the pressure $P_{0}$ corresponds, and $\tau_{\text {eq }}$ is the equivalent optical depth. Therefore, we have

$$
\begin{aligned}
\frac{\Delta f}{f}(\lambda) & =\frac{R_{\mathrm{pl}}^{2}+2 R_{\mathrm{pl}} H \ln \left(\frac{\epsilon_{\mathrm{abs}} \sigma_{\mathrm{abs}}(\lambda) P_{0}}{\tau_{\mathrm{eq}}} \sqrt{\frac{2 \pi R_{p} H}{k_{B}^{2} T_{p}^{2}}}\right)}{R_{\star}^{2}} \\
& =\frac{R_{\mathrm{pl}}^{2}+2 R_{\mathrm{pl}} H \cdot Z(\lambda)}{R_{\star}^{2}},
\end{aligned}
$$

where, for simplicity, we define

$$
Z(\lambda)=\ln \left(\frac{\epsilon_{\mathrm{abs}} \sigma_{\mathrm{abs}}(\lambda) P_{0}}{\tau_{\mathrm{eq}}} \sqrt{\frac{2 \pi R_{p} H}{k_{B}^{2} T_{p}^{2}}}\right) .
$$

Therefore, measuring $S_{\text {band }_{i}}$ in Equation (1) corresponds to computing the mean in the band:

$$
\begin{aligned}
S_{\text {band }_{i}} & =\left(\frac{\Delta f}{f}\right)_{\text {band }_{i}} \\
& =\frac{R_{\mathrm{pl}}^{2}}{R_{\star}^{2}}+\frac{2 R_{\mathrm{pl}} H}{R_{\star}^{2}} \cdot \frac{\sum_{j}^{M} Z_{j}}{M} \\
& =\frac{R_{\mathrm{pl}}^{2}}{R_{\star}^{2}}+\frac{2 R_{\mathrm{pl}} H}{R_{\star}^{2}} \cdot Z_{\text {band }_{i}},
\end{aligned}
$$

where $Z_{j}$ is the equivalent of Equation (A4) in the $j$ th spectral bin and $Z_{\text {band }_{i}}=\frac{\sum_{j}^{M} Z_{j}}{M}$. Therefore, the dispersion of Equation (2) is computed as

$$
\begin{aligned}
\sigma_{\text {band }_{i}} & =\frac{2 R_{\mathrm{pl}} H}{R_{\star}^{2}} \sqrt{\frac{\sum_{j}^{M}\left(Z_{j}-Z_{\mathrm{band}_{i}}\right)^{2}}{M}} \\
& =\frac{2 R_{\mathrm{pl}} H}{R_{\star}^{2}} \cdot \sigma_{Z_{\text {band }_{i}}},
\end{aligned}
$$

where $\sigma_{Z_{\text {band }_{i}}}=\sqrt{\frac{\sum_{j}^{M}\left(Z_{j}-Z_{\text {bandi }_{i}}\right)^{2}}{M}}$.

By combining the previous equations as done in Equation (3), we finally obtain

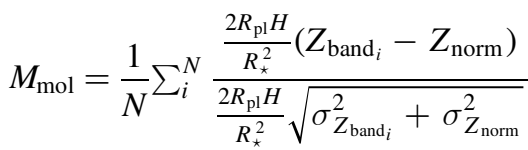

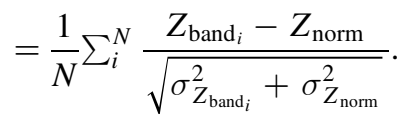

Therefore, we remove the planet and star radius dependence in the measurement. Similarly to what has been done in Désert et al. (2009), the subtraction between $Z_{\text {band }_{i}}$ and $Z_{\text {norm }}$ finally removes the scale height dependency as

$$
Z_{\text {band }_{i}}-Z_{\text {norm }}=\ln \left(\frac{\epsilon_{\text {abs, band }_{i}} \sigma_{\text {abs,band }_{i}}}{\epsilon_{\mathrm{abs}, \text { norm }} \sigma_{\mathrm{abs}, \text { norm }}}\right),
$$

where $\epsilon_{\mathrm{abs}, \text { band }_{i}} \sigma_{\mathrm{abs}, \text { band }_{i}}$ is the equivalent of $\epsilon_{\mathrm{abs}} \sigma_{\mathrm{abs}}(\lambda)$ in the band. This factor identifies the contribution of the main absorber in the band. Therefore, if we compare a band where a certain molecule has a strong feature with one where it is not supposed to give contributions to the spectrum, we can identify the molecular presence, compared to what is present in the second band.

So, finally $M_{\text {mol }}$ becomes

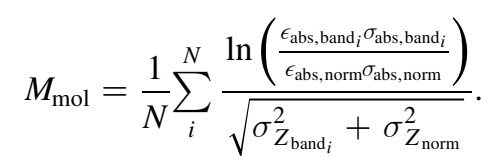

So, as promised, the metric is also sensitive to the molecular content.

To summarize, we removed the star, planet, and atmosphere size dependencies by subtracting the interesting feature bands for a normalization band and dividing the results by the combined dispersion. This results in a metric that is sensitive to the molecules contained in the atmosphere but introduces a bias. In fact, the spectral dispersion $\sigma_{Z_{\text {band }}}$ depends on both the atmospheric feature dispersion and the observational noise.

\section{ORCID iDs}

Lorenzo V. Mugnai iㅛ https://orcid.org/0000-0002-9007-9802 Ahmed Al-Refaie (1) https://orcid.org/0000-0003-2241-5330 Andrea Bocchieri (i) https://orcid.org/0000-0002-8846-7961 Quentin Changeat (1) https://orcid.org/0000-0001-6516-4493 Giovanna Tinetti i https://orcid.org/0000-0001-6058-6654

\section{References}

Abel, M., Frommhold, L., Li, X., \& Hunt, K. L. C. 2011, JPCA, 115, 6805 Abel, M., Frommhold, L., Li, X., \& Hunt, K. L. C. 2012, JChPh, 136, 044319 Al-Refaie, A. F., Changeat, Q., Waldmann, I. P., \& Tinetti, G. 2021, ApJ, 917, 37

Bakos, G. Á. 2018, Handbook of Exoplanets (Berlin: Springer), 111 Barstow, J. K., Changeat, Q., Garland, R., et al. 2020, MNRAS, 493, 4884 Barton, E. J., Hill, C., Yurchenko, S. N., et al. 2017, JQSRT, 187, 453 Caldas, A., Leconte, J., Selsis, F., et al. 2019, A\&A, 623, A161 Cessa, V., Beck, T., Benz, W., et al. 2017, Proc. SPIE, 10563, 105631L Changeat, Q., Al-Refaie, A., Mugnai, L. V., et al. 2020, AJ, 160, 80 Charbonneau, D., Allen, L. E., Megeath, S. T., et al. 2005, ApJ, 626, 523

Désert, J.-M., Lecavelier des Etangs, A., Hébrard, G., et al. 2009, ApJ, 699, 478

Edwards, B., Changeat, Q., Baeyens, R., et al. 2020, AJ, 160, 8

Edwards, B., Mugnai, L., Tinetti, G., Pascale, E., \& Sarkar, S. 2019, AJ, 157,242

Fletcher, L. N., Gustafsson, M., \& Orton, G. S. 2018, ApJS, 235, 24

Gaia Collaboration, Prusti, T., de Bruijne, J. H. J., et al. 2016, A\&A, 595, A1 Gandhi, S., \& Madhusudhan, N. 2017, MNRAS, 472, 2334

Goodfellow, I., Bengio, Y., \& Courville, A. 2016, Deep Learning (Cambridge, MA: MIT Press)

Guilluy, G., Gressier, A., Wright, S., et al. 2021, AJ, 161, 19

Hastie, T., Tibshirani, R., \& Friedman, J. 2009, The Elements of Statistical Learning (Springer Series in Statistics) (2nd edn; New York: Springer), 1

Hill, C., Yurchenko, S. N., \& Tennyson, J. 2013, Icar, 226, 1673

Hou Yip, K., Changeat, Q., Nikolaou, N., et al. 2021, AJ, 162, 195

Huitson, C. M., Sing, D. K., Vidal-Madjar, A., et al. 2012, mnras, 422, 2477 Irwin, P. G. J., Teanby, N. A., de Kok, R., et al. 2008, JQSRT, 109, 1136

Iyer, A. R., Swain, M. R., Zellem, R. T., et al. 2016, ApJ, 823, 109

Kreidberg, L., Bean, J. L., Désert, J.-M., et al. 2014, Natur, 505, 69 
Lecavelier Des Etangs, A., Pont, F., Vidal-Madjar, A., \& Sing, D. 2008, A\&A, 481, L83

Line, M. R., Wolf, A. S., Zhang, X., et al. 2013, APJ, 775, 137

Madhusudhan, N., Lee, K. K. M., \& Mousis, O. 2012, ApJL, 759, L40

Mayor, M., Pepe, F., Queloz, D., et al. 2003, Msngr, 114, 20

Moses, J. I., Visscher, C., Fortney, J. J., et al. 2011, ApJ, 737, 15

Mugnai, L. V., Modirrousta-Galian, D., Edwards, B., et al. 2021, AJ, 161, 284

Mugnai, L. V., Pascale, E., Edwards, B., Papageorgiou, A., \& Sarkar, S. 2020, ExA, 50, 303

Pedregosa, F., Varoquaux, G., Gramfort, A., et al. 2011, J. Mach. Learn. Res., 12,2825

Pepper, J., Stassun, K. G., \& Gaudi, B. S. 2018, Handbook of Exoplanets (Berlin: Springer), 128

Pluriel, W., Whiteford, N., Edwards, B., et al. 2020a, AJ, 160, 112

Pluriel, W., Zingales, T., Leconte, J., \& Parmentier, V. 2020b, A\&A, 636, A66

Pollacco, D., Skillen, I., Collier Cameron, A., et al. 2006, Ap\&SS, 304, 253

Polyansky, O. L., Kyuberis, A. A., Zobov, N. F., et al. 2018, MNRAS, 480, 2597

Rauer, H., Catala, C., Aerts, C., et al. 2014, ExA, 38, 249

Ricker, G. R., Vanderspek, R., Winn, J., et al. 2016, Proc. SPIE, 9904, 99042B
Rothman, L. S., Gordon, I. E., Barber, R. J., et al. 2010, JQSRT, 111, 2139 Seager, S., \& Sasselov, D. D. 2000, ApJ, 537, 916

Sing, D. K., Fortney, J. J., Nikolov, N., et al. 2016, Natur, 529, 59

Skaf, N., Bieger, M. F., Edwards, B., et al. 2020, AJ, 160, 109

Sturrock, G., Manry, B., \& Rafiqi, S. 2019, SMU Data Science Review, 2

Tennyson, J., \& Yurchenko, S. N. 2012, MNRAS, 425, 21

Tinetti, G., Drossart, P., Eccleston, P., et al. 2018, ExA, 46, 135

Tinetti, G., Encrenaz, T., \& Coustenis, A. 2013, A\&ARv, 21, 63

Tinetti, G., Vidal-Madjar, A., Liang, M.-C., et al. 2007, Natur, 448, 169

Tsiaras, A., Waldmann, I. P., Zingales, T., et al. 2018, AJ, 155, 156

Udalski, A., Szymański, M. K., \& Szymański, G. 2015, actaa, 65, 1

Waldmann, I. P., Rocchetto, M., Tinetti, G., et al. 2015a, ApJ, 813, 13

Waldmann, I. P., Tinetti, G., Rocchetto, M., et al. 2015b, ApJ, 802, 107

Wheatley, P. J., Pollacco, D. L., Queloz, D., et al. 2013, in EPJ Web of Conf. 47, Hot Planets and Cool Stars Vol. 47, ed. R. Saglia (Garching: EPJ), 13002

Yurchenko, S. N., Barber, R. J., \& Tennyson, J. 2011, MNRAS, 413, 1828 Yurchenko, S. N., \& Tennyson, J. 2014, MNRAS, 440, 1649

Zellem, R. T., Swain, M. R., Cowan, N. B., et al. 2019, PASP, 131, 094401 